\title{
LA PERPLEXITÉ DE L'AUTOBIOGRAPHIE DANS \\ LE JARDIN DE BADALPOUR \\ DE KENIZÉ MOURAD
}

\author{
Dr. Amani Samir \\ Professeur-Adjoint \\ A la Faculté des Lettres - Université de Benha
}

\begin{abstract}
Résumé:
L'autobiographie est un genre littéraire qui relève de la littérature intime ; la littérature de soi: La nouveauté qu'apporte Kenizé Mourad dans le Jardin de Badalpour est différente ; car elle crée une perplexité dans l'écriture. Le lecteur se trouve dans un labyrinthe contradictoire de mémoire et de souvenirs accumulés entre réel et fictif. L'auteure- narratrice et son personnage central "Zahr" sont confondues en une seule personne qui raconte selon un "je" et une "elle" de telle sorte que l'imagination s'intègre à travers la réalité.

Le but de la recherche réside dans cette contradiction perplexe entre un "je" réel et un "elle - " "je" fictif. Nous démontrerons à travers plusieurs séquences que l'auteure-narratrice trace un itinéraire de cette quête de soi; perdue dans de nombreuses identités; cherchant son origine et ramenant dans son fort- intérieur des espaces divers de l'origine de l'homme blessé d'injustice sociale, politique et familiale, se contredisant entre des sentiments d'amour et de haine envers un père Radja qui a délaissé sa fille pour être adoptive de certaines familles américaine, suisse, française. "Zahr" ou la narratrice dénonce cette relation, évoque les moments de bonheur et de malheurs envers sa mère morte Kadija descendante de la dynastie ottomane et à travers le jardin en Inde auquel elle aspire nostalgiquement de la Turquie jusqu'aux Indes; Kenizé Mourad nous trace une fresque panoramique de toute l'humanité occidentale et orientale pour nous reléguer un message de paix et de Bonheur. Elle se vide pour se recréer de nouveau.
\end{abstract}

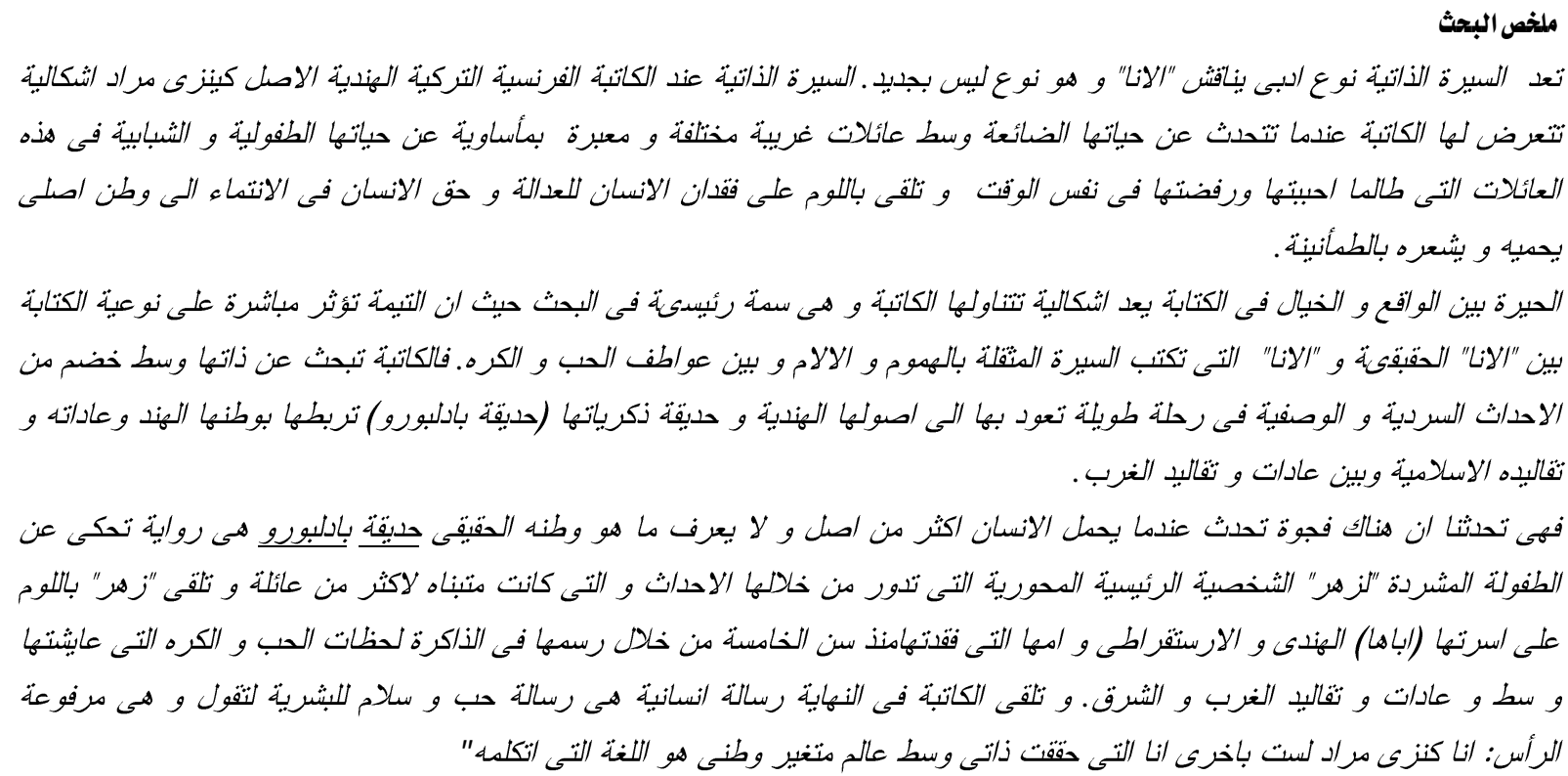

Biographie et renseignements sur Kenizé Mourad

Kenizé Mourad, écrivaine française, née à Paris en 1939, romancière et journaliste française d'origine turco-indienne. Elle a reçu le grand prix des lectrices (Elle) Roman en 1988.

Kenizé Mourad est la fille d'une princesse turque, descendante du Sultan Mourad V par sa mère, Hatidjé Sultane, mariée à un rajah indien, mais refugiée à Paris.
Peu après sa naissance, sa mère meurt, elle la déplore dans son roman De la part de la princesse morte. Elevée, dans un milieu catholique, orpheline qui fut éduquée dans un institut religieux ; elle découvre à l'âge de 20 ans l'Islam dans les textes des grands Soufis. Elle conçoit l'Islam comme une religion ouverte et tolérante. Auteure de 7 romans :

1- De la part de la princesse morte,

2- Le jardin de Badalpour, 
3- Dans la ville d'or et d'argent,

4- Le parfum de notre terre,

5- A la rencontre des Maharanis, reines et princesses indiennes (12 livres),

6- Turquie (9 livres),

7- Nostalgie et splendeur au pays d'Ataturk (26 livres).

Parmi ses amis proches nous citons : Michel de Grèce, Irène Frain, Fanny Descharny, Régine Deforges, Amin Maalouf.

Kenizé Mourad fait des études de sociologie, et de psychologie à la Sorbonne avant d'être journaliste. En 1970, elle rejoint l'équipe du Nouvel Observateur sous le titre de grand reporter du Moyen-Orient, et du sous continent indien, où elle couvre entre autres, les révolutions iranienne et éthiopienne, la guerre du Liban, effectue de longs séjours en Inde et au Pakistan. En 1983, elle se consacre à l'écriture. Elle vit maintenant en Irlande.

De la part de la princesse morte est un célèbre roman qui connut un tirage de plusieurs millions d'exemplaires. Une vraie saga familiale dont le jardin de Badalpour constitue le deuxième volet.

\section{Avant-propos}

Un départ à la recherche du passé, afin de reconstruire sa propre vie. Tel est le défi que lance K. Mourad pour écrire et raconter son propre histoire. Le jardin de Badalpour est un chef-d'œuvre qui appartient au patrimoine littéraire et mondiale, et qui prône avant tout la dignité de l'être humain.

Narration, description des lieux divers; discours; longues dissertations, romantisme, philosophie esthétique, et techniques d'écriture, le tout se combine et s'harmonise dans un roman plein d'émotion et de fidélité, d'authenticité et de réflexions sur la condition de l'être humain, sur le statut de la femme orientale, son amour, ses droits, son émancipation, son enfermement et sa liberté ; sur le statut des enfants délaissés et orphelins. Roman autobiographique, le jardin de Badalpour lance un grand cri d'appel à la conscience humaine pour se ressaisir.
L'écriture pour K. Mourad est un perfectionnisme d'artiste qui cherche les moindres détails. L'écriture lui paraît un investissement. "J'ai besoin de temps pour écrire; je suis perfectionniste, je vérifie les moindres détails (...) par ailleurs, j'ai besoin de m'isoler, de récréer tout un monde; de me construire une bulle (...) Après j'ai besoin de respirer, de vivre (...) je me suis rendu compte que je ne pouvais plus écrire si je ne m'attaquais pas à mon histoire personnelle. ${ }^{1}$

Le roman, depuis ses origines, n'a cessé de jouer avec le lecteur un jeu de masquage de la vérité et du mensonge. Lorsque l'auteurécrivain s'autocite, le récit revêt un caractère autobiographique apportant une émotion particulière, et une résonance différente à la lecture. L'auteur-écrivain exalte presque tous les détails minimes de sa propre vie et utilise un matériau authentique sans se déguiser.

C'est toujours une écrivaine qui parle, énonce sans ambiguïté et sans confusion son œuvre autoréflexive. Le jardin de Badalpour de Kenizé Mourad offre au lecteur un patrimoine riche d'indications et d'informations inconnues cachées dans un for-intérieur, sous-jacent d'une volonté de système.

La légitimité de cette approche consiste à relier l'autobiographie de l'auteur à des notions conçues, à des rituels de conditions familiales, sociales, et à un système de lecture rétrospective. C'est pour cette cause, que le roman apparait dans toute sa complexité la plus profonde, une sorte de « constellation ${ }^{2}$.

C'est ainsi que nous tenterons de justifier, tout au long de notre travail le processus d'une écriture autobiographique qui relève de la problématique de l'expérience personnelle. «Je n'écris pas par amour de beau style (...) j'écris pour faire passer des idées (...) que le vrai islam est un islam ouvert et modéré ; que le statut des femmes n'a pas toujours été celui que l'extension actuelle du niqab laisse imaginer la morgue 
occidentale, tout comme le racisme, sont fondés sur l'ignorance de l'autre $»^{3}$

Notre objectif fondamental sera le déchiffrement de cette perplexité à travers une étude ayant pour essence les problèmes posés entre :

auteur-lecteur-narrateur / auteur-lecteurécrivain

dont nous aborderons ici l'étude. A ce propos, il convient donc de citer les mots de Walter. Benjamin ${ }^{4}$ : «J'appris de bonne heure à me dissimuler dans les mots, qui étaient en réalité des nuages. Le don de reconnaître des similitudes n'est, en effet, rien d'autre que les vestiges affaiblis de la vieille compulsion à devenir semblables aux autres, à se conduire comme eux».

L'imagination autobiographique pour $\mathrm{k}$. Mourad est une faculté de comparaison, de formation, de combinaison, de discrimination, de représentation non perspective. Elle est d'emblée une expérience transcendantale.

Dans ce récit passionnant et fascinant, $K$. Mourad se dévoile en disant:«J'ai voulu raconter cette histoire. J'ai voulu raconter un autre islam. J'ai voulu montrer que les religions disaient la même chose par des chemins différents et lorsqu'elles s'entendaient, cela produisait des merveilles $\gg$.

«Mon domaine, c'était les pays de la sphère musulmane et du sous-contient indien, et je voulais expliquer aux Français des choses auxquelles ils ne comprenaient rien $\gg{ }^{6}$

Etablissant un contrat ou un pacte de lecture sans tomber dans le piège de faire de son histoire raconté un mythe à suivre; elle rejoint Philippe Lejeune dans son œuvre, Moi aussi quand il écrit: «Le terme "contrat" suggère qu'il s'agit de règles explicites, fixes et reconnues d'un commun accord par les auteurs et par les lecteurs: chez le notaire, les deux parties signent le même contrat, au même instant. Rien de tel en littérature» ${ }^{7}$

\section{Introduction}

L'autobiographie, journal intime, écriture de soi, pamphlet; écriture ratée tire son étymologie d'une définition grecque: Ecrire sa propre vie (soi) et (graphie) de (graphein) et (écriture) et de bios (vie).

Au sens large, l'autobiographie doit contenir un minimum de vérité lié avec un minimum d'illusion, de fantasmes, de rêves et d'imagination. Ce mot apparait au XIX ${ }^{\text {ème }}$ siècle à peu près au environ de 1832-1842.

Les précurseurs de ce genre sont multiples; nous avons retenu quelques noms comme J.P. Sartre (Les mots), (Le livre de ma mère) d'Albert Cohen, Le monde d'hier : souvenirs d'un européen, Stephan Zweig. (L'Écriture ou la vie) de Jorge Semprun, (Pour l'amour de l'Inde) de Catherine Clément, ( de tous les miens) de Martin Gray, (Les confessions) de J.J. Rousseau, (Mémoires d'une jeune fille rangée) de Simone de Beauvoir; (Une vie) de Simone Veil, (Enfance) de Nathalie Sarraute; (Ma vie) de Carl Gustave Jung. (L'âge d'homme) de Michel Leiris; (Ma vie rebelle) Ayaan Hirsi Ali, (Dans ma peau), (autobiographie) de Doris Lessing et bien d'autres; la liste est continuelle jusqu' à nos jours. L'autobiographie est un terrain fécond qui ne tarit jamais.

L'on pourrait dresser une typologie de ces confidences en passant par M. Yourcenar Carnets de notes des Mémoires d'Hadrien, donnés en postface au roman), Paul Ricœur, Les sens d'une vie, Paris, ed. La découverte,1997, Nathalie Rheims, Journal intime, Léo Sheer, 2007.

Kenizé Mourad s'octroie un récit autobiographique original qui s'ouvre à contre- courant de tout récit autobiographique. Ce qui constitue une duplication paradoxale de l'origine de l'histoire racontée. Ecrire son autobiographie c'est choisir le commencement et la fin ; or k. Mourad écrit pour en finir, pour traiter son écriture comme sa propre fin. L'auteure en se racontant photographiant ses souvenirs et sa mémoire, décide de s'en délivrer. C'est dans cette contradiction sémantique de l'origine que ce récit autobiographique trouve son sens. Se contentant d'évoquer rapidement 
dans le projet qu'elle s'octroie, elle détourne la fonction, le rôle et enjeux esthétiques d'une écriture qui édifie le "soi".

Vouloir remonter à ses propres origines est une vertu littéraire, une mise en œuvre d'une controverse perplexe.

De façon plus ou moins avouée, le rêve et la hantise de la postérité produisent une parole qui trouvera son efficacité, l'instant d'après.

Ainsi le récit autobiographique de l'auteure est centré sur une période et un lieu précis unifiés par les motifs de l'enfance, l'adolescence marquant des étapes de vie désordonnées et chaotiques d'un traumatisme irrémédiable.

Le fait de s'écrire, c'est rendre son écriture transitive, transformable dans le processus qu'elle transfigure et la motivation d'une tension significative. L'auteure parvient à une résolution lucide: l'autobiographie est une volonté d'écrire non de se justifier.

Considérons de plus prés, la structure narrative dans cette autobiographie : ce qui attire l'attention, dès la première lecture au départ; c'est la défaite et l'échec du personnage qui baigne dans le malheur; d'une naissance dite "bâtarde" ; la période de l'enfance douteuse de son entourage qui subit une crise détruisante, et un revirement définitif enfin de compte de la situation qui précipite le protagoniste à la catastrophe finale.

«Avant tout tenté pour me le faire oublier, (...) Mon père, mon père indien me demande! Il n'a pas oublié sa fille! Bien sûr que je veux retrouver mon père, et tout de suite! (...) «papa, papa » savourant ce mot merveilleux (...) je n'étais plus une enfant abandonnée, j'avais un merveilleux papa: il m'aimait! $\gg{ }^{8}$

Cette transformation de la structure rend inévitable la fin tragique. D'autre part, par un processus inconscient de projection, le lecteur participe au mystère et au drame. Il a le sentiment d'une personnalité qui s'entraîne dans une action héroïque - quant au personnage il subit un destin extraordinaire.
Le lecteur est introduit dans un monde ludique, à travers l'interprétation cosmique, imprégné par le caractère psychique individuel; celui de la narrative "Zahr" qui confronte le monde.

L'autobiographie chez kenizé Mourad dépasse les frontières indiennes pour se lancer vers un humanisme mondial.

- C'est à travers l'œuvre autobiographique qu'on peut être témoin de l'émergence d'une nouvelle phase d'écriture dans ce domaine.

Genre ancien ou nouveau, l'autobiographie de kenizé Mourad ouvre une nouvelle ère de l'écriture spontanée et automatique.

Riche en pluralité de formes d'écriture; riche en polyvalence de symboles, l'écriture de K. Mourad est un instrument de la connaissance mutilée.

Cette écriture a pour fonction de montrer l'intrinsèque quotidien de l'enfant abandonné.

Dans le récit autobiographie de K. Mourad, il n'y a jamais de degré zéro, le récit est surchargé de symbole, des métaphores de l'intertextualité; de figures ou configurations symboliques qui penchent presque vers le récit mythique qui ne propose pas une certitude mais une hypothèse possible.

C'est pourquoi l'écriture autobiographique qui prend racine dans les tréfonds vécus des conjonctions, et qui traduisent en grande sorte, les relations humaines en allant de pairs avec les structures sémantiques.

Ce phénomène complexe qui relève du contexte social contribue à présenter un caractère assez singulier.

L'écriture est riche en polyvalence et ne se réduit jamais à une seule interprétation sans être mutilée. L'écriture autobiographique de K. Mourad est un instrument efficace de connaissance et en relation directe avec la condition humaine.

Comportement de la psyché; l'interprétation $\mathrm{du}$ fait quotidien individuel de Zahr n'est que la propre vision de l'auteure. Zahr subit un destin fatal; extraordinaire. Le monde dans lequel elle vit, s'écroule à la fin. Ce 
monde qui englobe des personnages secondaires, fictifs ou réels, anonyme ou anodins, encadre le malheur de sa vie. Tout un monde ludique survit à travers Zahr ayant même structure familiale (père - mère - enfants - sœurs et frères) et peu d'entre eux affectent et influent la vie de Zahr.

Ex: la maîtresse, la mère des religieuses ... C'est un monde d'un espace temporel et selon les propos de Gérard Genette : «l'espace peut être en relation directe avec la littérature dans ses rapports avec le monde».

Dance ce monde; le lecteur est transposé en imagination dans des lieux communs et dans des pays existants; exotiques parfois comme les Etats-Unis, la Suisse, la France mais cela ne dure qu'un bref épisode pour reprendre à nouveau un retour à la narration de l'histoire. Il nous semble que l'espace est mieux exprimé à travers le langage symbolique; fictif et réel.

L'autobiographie de K. Mourad à travers son personnage-héros constitue un champ d'investigation, un questionnement incessant sur la condition humaine et une réflexion sur la forme esthétique.

K. Mourad a su faire de l'autobiographie une symbolisation réflexive tout en gardant le mouvement ascendant du récit romanesque. Perspective spécifique, rapport littéraire et même philosophique s'inscrivent chez k. Mourad dans le cadre d'études de réception.

Considéré, comme un discours intime mais étranger an sien; l'élément philosophique émerge du récit comme une référence culturelle entre la confrontation de deux civilisations opposées: l'une représente l'Inde musulmane, l'autre, l'Europe occidentale chrétienne par une flexibilité habile de l'auteure, elle a su utiliser l'histoire de la vie de Zahr à concilier ce rapport opposé entre personnages, civilisation et contraintes sociales.

Pendant plusieurs pages et un nombre infini d'allusion, le texte cède le pas de la narration pour devenir un discours moralisateur. K. Mourad refuse de donner un sens unifié et global à son texte alors, le passé s'anéantit, le futur reste mystérieux, et seul compte le présent avec tout ses contraintes.

La quête de l'origine devient polysémique : image et figuration, écriture, titre et récit, description, témoins, spécularité et mise en abyme. Ce n'est pas la naissance qui fait l'affaire mais le texte littéraire qui la remplace donnant à l'objet décrit une fonction poétique sans conteste, où le destin d'écriture et héritage familial reviennent au même. Enfin, il nous convient de dire que l'autobiographie de Kenizé Mourad est « une anamnèse du soi par le "je" pour devenir "moi" 10

\section{Ch.I (1-1) La perplexité de l'écriture et les différents modes}

Grande œuvre, parsemée d'analyses perspicaces et sincères dans un récit «du vécu », où l'écrivain nous esquisse son épopée familiale avec les mémoires de Zahr, ${ }^{11}$ fille de la princesse Selma, petite fille de la sultane Khadidjé. Elle poursuit une quête discontinue dans un labyrinthe de puzzle. Reconstituant son passé par bribes; elle part à la découverte de sa famille surtout après la mort de sa vraie mère. « $\underline{\mathrm{De}}$ la part de la princesse morte ${ }^{12}$ est un roman qui précède Le jardin de Badalpour. Ecrit avec beaucoup de lucidité et de finesse féminine, K. Mourad nous entraine dans son itinéraire du quartier latin à Paris des années 60 au mystérieux jardin de Badalpour. Plusieurs familles l'accueillent d'origine différente où elle cherche amour et affection. Adolescente, étudiante, mariée elle comprendra que dans une société musulmane, le mariage est un contrat légal établi entre deux familles, deux fortunes, deux positions, ce contrat est sacré et forme la base de structure de société.

Interdisant toute forme publique, la femme mariée chez les orientaux n'est qu'une incongruité paradoxale. En enfermant les femmes dans le carcan des tabous dogmatiques, c'est à eux qu'ils font la plus grande erreur car la narratrice se demande-telle. Comment une mère ignorante du 
monde peut-elle former son fils à affronter la réalité ? Comment une jeune adolescente musulmane doit-elle se convertir au christianisme sans le vouloir? Comment jouir dans un monde occidental différent d'elle-même ? Dès lors le fils portera toujours le sentiment de rébellion.

A quinze ans, l'héroïne découvre qu'elle a tout perdu : ses parents, son nom, son pays, elle ne sait presque rien. A 21 ans, à l'âge de maturité, elle prend son courage à deux mains et décide d'aller en Inde pour rencontrer son père et sa famille. Arrivant trop tard, elle subit le choc de sa mort et doit confronter une famille dont elle ignore les attitudes et les comportements. Entre sentiments de haine et d'amour, l'auteure écrivaine nous expose des tableaux qui racontent sa relation avec son père, son frère et les problèmes des femmes dans la société indienne. Parvient-elle à trouver son identité perdue ou restera-t-elle toujours en quête comblée de vide et de néant.

$K$. Mourad nous plonge dans un océan de sentiments flagrants, fascinants dans les cultures de l'Inde dans une préhistorique et posthistorique de l'indépendance de l'Inde.

$\mathrm{La}$ représentation du «moi » est un sentiment subjectif caché, doué de puissance, et d'énergie intérieure. L'homme s'est toujours préoccupé de ce moi enfoui qui s'est exprimé dans le mythe du Paradis perdu et de la chute de l'homme depuis Platon. Ce moi immédiat et concret lutte contre le rejet de la réalité virtuelle.

La théorie de Freud sur l'existence d'un moi intérieur spontané fut l'énoncé le plus puissant dans les sociétés réprimées. Les psychanalystes l'ont idéalisé en le faisant le vrai fondement authentique, d'une plus grande valeur. $\mathrm{Ce}$ « moi » est en état de lutte constante de tension à l'infini, d'un déséquilibre perpétuel en rapport avec nos instincts, ce «moi » refoulé contre les pulsions. En dépit de la diversité des approches des psychanalystes qui ont donné à «l'id »une place prépondérante. Norman O. Brown ${ }^{13}$ selon son raisonnement passionnant, croit que nous pouvons dépasser notre humanité par la négation des pulsions de l'ego conscient, par un culte constant de la pulsion spontanée, pour trouver une humanité nouvelle et authentique, dans une action dirigée par une pulsion polymorphe continue. En généralité, Marcuse, nous révèle que les aspects du psychisme individuel qui contrôlent la pulsion sont devenus des forces répressives de la société.

Même si l'individu connaît sa propre oppression, son propre psychisme (le superego) est en complice avec cette force répressive. L'expression des pulsions doit être source de libération.

Selon Laing, il n'y a pas de réalité en dehors de l'expérience individuelle. $\mathrm{Ce}$ matérialisme n'a de continuité que dans la conscience. Personne ne peut vraiment savoir ce que ressent l'autre.

Les perceptions que nous avons de nous même et du monde ne peuvent pas être tolérés comme marginales. «Laing, avec la perception propre au romancier, a délimité les forces par lesquelles la société en général, les familles ou les individus en relation les uns avec les autres pour les mettre dans un moule et leur enlever toute valeur ». ${ }^{14}$ Même si, les sociétés obscurantistes et oppressives donnent l'apparence d'être tolérantes aux déviations des individus, nous plongeons de nos valeurs superficielles qui se prétendent rationnelles. Les hommes incapables d'être des marginaux prouvent un sentiment offensive contre les criminels, les fous, les débiles qui sont excommuniés, retirés de la vue du public. Nous vivons dans une société dans laquelle l'empirisme est une véritable éthique.

Dans cette perspective, l'individu constitue une révolution menaçante de la structure toute entière de la société.

Il est évident que la psychiatrie a fait du «moi » libre, spontané son favori de recherche et de préoccupation. Ceux qui perdent tout contrôle d'eux-mêmes se tiennent à l'écart c'est pourquoi, ils manifestent l'anorexie, l'auto-mutilation, le 
suicide à plus forte raison; s'ils vivent avec le monde, ils sont des êtres angoissés et névrosés et les conséquences deviendront de plus en plus dangereuses.

Pour donner à cette approche une existence valable et pour plus d'efficacité, il s'avère que Kenizé Mourad intègre ce «moi» profond dans son personnage pour qu'on puisse le comprendre et l'apprécier; il faut l'éloigner de la «marginalité ». Cet état mérite toute notre attention pour être justifié par tous les moyens et les procédés d'écriture.

Que peut apporter la lecture de ces romans à un lecteur du XXI ${ }^{\text {ème }}$ siècle ? Seraient - ce les premiers signes d'un retour à soi ?

Cette méditation sur l'égocentrisme est riche d'informations cognitives que nous livre l'écrivaine. Des intentions fulgurantes de romancière sur ce mystère qui exploite le «moi qui est un autre ${ }^{15}$ qui n'en finit pas de heurter les esprits des ses lecteurs. En parcourant tout le roman de K.Mourad, nous partageons son cri d'effroi, d'incompréhension, de souffrance dans sa quête sur «l'origine ».

Nous suivrons, étape par étape, l'évolution des sentiments présentés d'un personnage traqué par le mal involontaire d'être adoptif dans plusieurs familles. (D'ailleurs; K. Mourad est d'origine mixte). Comment cette romancière a pu composer et recomposer son statut personnel et son corpus personnel dans une tradition qui refuse les valeurs humaines? (rappel constant dans la trame du roman.) Cette idée de l'anonymat marginalisé au sein d'une société qui combine le rituel occidental avec le rituel oriental depuis l'acte de naissance en passant par la jeunesse, et en arrivant à la vieillesse ou en d'autres termes de l'alpha à l'oméga.

Les commentaires faits par K. Mourad n'appartiennent donc pas à une narratrice extérieure à l'histoire, puisque c'est son histoire de vie qu'elle raconte. C'est pourquoi elle fait appel à des énoncés de modalité appréciative et affective. $\mathrm{Sa}$ subjectivité dans les récits s'impose remarquablement dans les types de narration qu'elle choisisse.

Le récit relatant toute la période de l'enfance; l'éducation religieuse qu'elle a reçue ; toute cette partie qui débute l'œuvre est racontée à la première personne du singulier.

Dans cette partie la narratrice est le personnage principal : je cite

«Je ne veux plus voir personne, je veux m'enfouir au plus profond de cette terre, je veux mourir. (...) depuis trois ans que je me rongeais, mais ma souffrance importait peu... ». ${ }^{16}$

Récit rétrospectif qui raconte l'origine de la narratrice. Il s'agit d'une prolepse à l'intérieur du récit rétrospectif.

Donc revenant à l'implication directe de la narratrice; elle se voit par le biais du pronom personnel «je»: singulier qui se transforme au cours de l'histoire chronologiquement en «nous» pluriel et parfois un «on» impersonnel (parlant de la communauté des religieuses - les parents ou les collègues et amis. "Tous devinrent ou restèrent " des intellectuels grandsbourgeois" (...) mon professeur Dorlin (...) notre chef Ménéchal. Parmi ces intellectuels, il y avait là deux ouvriers que le groupe avait réussi à récupérer l'un d'eux finit par nous quitter. ${ }^{17}$

La narratrice débute son récit in média-res.

Effet de dédoublement de la narratrice homodiégétique - dualité énonciative du « je $»$ :

Dans le jardin de Badalpour, les événements racontés s'intègrent selon le point de vue de la jeune narratrice âgé de 15 à 17 ans qui retourne à son pays natal aux Indes pour assister aux funérailles de son vrai père (je souligne vrai père) parce qu'en faite; la narratrice possède plusieurs pères; fille adoptive de plusieurs familles.

A ce propos René Rivara nous indique que le narrateur autobiographe se dédouble en deux instances : " D'une part, nous sommes en présence d'un "narrateur -scripteur" ou "narrateur- narrant" qui sait tout de sa propre histoire, et même, éventuellement de 
ce qui s'est passé entre la fin de l'histoire et le moment où il écrit, et qui a le pouvoir d'intervenir dans le récit de son passé, muni des connaissances acquises après la période de sa vie qu'il raconte; et de l'autre, un "narrateur-personnage" dont la vie, les aventures, les sentiments, l'activité nous sont contés et qui fait partie intégrante de la diégèse (l'histoire) ». ${ }^{18}$

Il se dégage de là : qu'on a en effet deux narrateurs: le narrateur-narrant qui reste toujours compatible avec le temps du discours employant le présent de l'indicatif et un narrateur-scripteur qui dénote tous les personnages de l'histoire, hormis le personnage qu'il était lui-même dans le passé d'après Rivara René ${ }^{19}$ déjà cité.

Le "je" du narrateur-personnage est régulièrement associé aux temps de l'énonciation historique (imparfait-plus que parfait -passé simple). En revanche le narrateur-scripteur est associé aux temps de l'énonciation discursive (le présent-le futur et le passé composé) ; il correspond donc à l'énonciateur originaire du récit. " Moi aussi, j'étais une militante / atypique, je n'avais jamais lu et n'avais aucune intention de lire Marx, Lenine ou Trotski (...) J'étais, sans le savoir de la vraie graine de révolutionnaire $» .^{20}$

C'est pourquoi Rivara ajoute que « Dans un récit autobiographique, le personnage, en tant qu'il peut se distinguer du scripteur, est donc, assez naturellement pourvu, aux différents moments de l'histoire, d'un point de vue, qui peut être district ou non de celui du scripteur. ${ }^{21}$ J'ajoute que M. Bakhtine met aussi l'accent sur le rôle du narrateur homodiégétique en disant: « Si je narre, (ou relate par écrit) un événement qui vient de m'arriver, je me trouve déjà, comme narrateur, hors du temps et de l'espace où l'épisode a eu lieu ${ }^{22}{ }^{22}$

Ainsi, pour prouver le dédoublement du narrateur : Zahr dit : "Comment aurais-je pu élaborer calmement des hypothèses et découvrir cette vérité dont j'avais l'impression que toute ma vie dépendait $\gg .{ }^{23}$
Dans la première instance narrative, on a une narratrice adulte qui jette un regard rétrospectif sur son passé. Ainsi, le pronom «je » s'engage dans une double fonction: en tant que le " je-narrant", il assume la narration du récit et en tant que le " jenarré", elle remplit son rôle d'un personnage dans l'histoire.

Dans d'autre scène le narrateur- enfant qui fait partie essentielle de l'histoire racontée assume la responsabilité de la totalité du récit ou en d'autres termes l'énonciateur de tout le récit homodiégétique telle que l'affirme Philippe Lejeune : «C'est la voix du narrateur adulte qui domine et organise le texte : s'il met en scène la perspective de l'enfant, il ne lui laisse guère la parole. C'est là bien naturel : l'enfance n'apparaît qu'à travers la mémoire de l'adulte. ${ }^{24}$

Prenant aussi le point de vue de Vincent Jouve le terme « focalisation » est défini comme « la restriction de champ - ou plus précisément la sélection de l'information narrative. $»^{25}$

Quant aux personnages; la narratrice se contente d'enregistrer du dehors les comportements et les actions de ses personnages sans aucun accès à leurs pensées et leurs sentiments. Elle met tout l'accent à la focalisation externe « excluant par là toute possibilité d'information sur les pensées de quiconque. $\gg^{26}$

Ainsi toute appréciation ou interprétation subjective est exclue. C'est pourquoi dans l'autobiographie; le récit devient subjectif et la narratrice elle-même sera « un sujet fictif de toutes les perceptions y compris celles qui la concernent elle-même comme objet. Tous les événements sont présentés à travers le regard, les émotions et la sensibilité de la narratrice Kenizé Mourad. Il s'avère important de souligner que dans l'autobiographie, la narration se présente avec simultanéité ; la narratrice raconte le monde dans l'instant même où elle semble en faire l'épreuve.

La subjectivité du récit autobiographique apparaît «à tout phénomène qui caractérise un individu en tant qu'il est unique. Les 
sentiments qu'éprouve un être humain, ses comportements, ses jugements, dans l'appréciation d'une situation complexe, le caractérisent en tant que tel et sont, en ce sens, " subjectifs". » ${ }^{27}$

Le récit autobiographique de K. Mourad est bâti sur une alternance régulière entre des plans larges et des plans centrés sur les familles de la princesse. K. Mourad construit une fresque historique à la manière de Tolstoï en mêlant des destins individuels. Les choix qu'elle s'approprie influent beaucoup sur la trame événementielle. L'humanité suit un parcours écrit à l'avance qui la dépasse. Les événements racontés marquent le début d'une grande dépression de colonialisme britannique sur l'Inde. Une crise de perte de confiance durable à l'égard des systèmes politiques. La colère que la romancière éprouve, dénonce la société contemporaine amputée de la grâce divine et donne privilège aux puissances humaines et rattache une espérance de salut et de libération. Ainsi, elle en fait de ce constat, le cri insupportable des captifs qui demandent la délivrance et veulent obtenir la justice. Ces divers aspects reflètent en quelque sorte un patronyme familial issu d'un rituel d'habitudes, de coutumes, de normes traditionnelles contre lesquelles, elle se révolte obstinément.

Fille adoptive par nature, la narratrice évoque les rapports de garder une famille unie. Sans perdre la foi au travers des épreuves. Pour exprimer la force de la Foi, la narratrice succombe aux assauts du mal et s'abrite contre les duretés du monde.

A la fin du roman, K. Mourad se distingue par sa douceur; son altruisme; par son retour de l'enfant prodigue sur la terre ingrate.

Euvre intemporelle qui transcende le temps par son parcours initiatique; par son drame humanitaire; sa monstruosité cruelle, elle nous confie avec cœur navré, et selon une logique simpliste et affective; pour qui elle doit payer le forfait de son existence.

Ce roman est bien le récit d'une injustice sociale dont il convient d'analyser la complexité subtile des plans proposés.
Contre tout ordre humain malmené; $\mathrm{K}$. Mourad vit dans une communauté indienne puis immigre vers l'occident et retourne à son pays natal lors des funérailles de son père le prince. Pour survivre elle accepte, bon gré mal gré, le statut involontaire d'être adoptive de certaines familles. Expulsée, elle éprouve le sentiment d'être arracher de sa racine; elle était obligée, donc, de se soumettre à un ordre destructif malgré les affections et l'amour qu'on lui a donnés. Contraintes inévitables; horreur de situation ; elle remet la cause au destin. Sans le savoir, elle se met à réécrire à sa manière un drame de désobéissance à un monde hostile ou inconnu qui ne tarde pas à lui émettre un ensemble de règles régies par la vie en société. C'est ainsi que la narratrice définit les espaces privés des communautés parmi lesquelles elle mènera le prix des tabous à ne pas enfreindre. Le savoir-vivre, les fêtes, les funérailles recréent et avivent sa mémoire personnelle. Aussi substitue-telle un corpus de lois non- écrites aux lois familiales. Ses règles ne sont pas plus justes, mais présentent un immense avantage d'adéquation pour assurer la cohésion sociale.

Une autre caractéristique vient s'imposer au corpus édifié. C'est sa capacité volontaire d'adaptation raisonnable sorte de "contrat social" $^{28}$ ou pacte pour assurer le bien commun et l'intériorisation des interdits. L'unité familiale constitue le noyau minimal dont l'écrivaine aspire.

Comme femme écrivaine, K. Mourad lance un cri d'amertume et de remords d'une jeune fille abandonnée à elle-même, cherchant sa propre autonomie.

Ch.I (1-2) Un langage métatextuel entre réel et fictif

Le langage qui permet de s'exprimer et d'entrer en communication véhicule, non seulement, par l'appartenance à une classe sociale, mais encore par des concepts culturels. La narratrice se forge un langage particulier qui s'articule sur les sens; le regard, les sentiments farouches du cœur; les sentiments des moments de bonheur; et 
privilégie la sensation de la vie tout en s'exprimant par le " je" par excellence.

Bien qu'elle est issue d'une famille riche, elle utilise un langage qui rejette l'hypocrisie et en cherche la sincérité moralisatrice. Par la connotation des forces de mots employés; elle réussit à en donner une expérience personnelle authentique.

- La quête d'un paradis familial perdu au bout d'un chemin pigmenté d'épreuves, reste le ressort de cette histoire. Vibrant appel à la société, Le jardin de Badalpour reste une œuvre d'actualité digne d'un grand salut.

De l'autobiographie à l'autofiction, le rôle de l'imagination est seul apte à retracer toute la profondeur de l'expérience de la narratrice : Une expérience aventureuse, renouvelée et mouvante. La confrontation de la réalité se heurte avec le fictif qui envahit le langage de l'intime chez K.Mourad.

Dans La relation critique de Jean Starobinski qui définit le langage où $[\ldots .$. « l'expression procède de l'expérience, sans discontinuité aucune, comme la fleur résulte de la poussée de la sève ou du jet de la tige $\gg .29$

Nous envisageons ici une nouvelle approche du réel que celle-ci induit. En dépit des apparences, le style que s'approprie K. Mourad forme un «écart». Cet «écart» désigne cette transgression spontanée du code et de la norme. L'œuvre s'avère une «poétique» d'un type autobiographique nouveau qui esquisse la fidélité des mots aux choses vécues.

L'autobiographie de K. Mourad paraît ainsi « un plaisir d'écrire » développe une théorie de sa propre écriture en train de se faire écho d'une réalité amère, une vérité subjective qui se désintéresse de la vérité factuelle. Le style de l'écrivaine paraît alors comme «un chaos incompréhensible» plausible à l'autofiction. Entre autobiographie et autofiction, il s'avère qu'un conflit se restitue d'une intensité inouïe quand l'écrivaine s'oppose à ellemême. Quand la fiction honteuse passe à une fiction triomphante.
En ceci, K. Mourad comme le suggère, Gérard Genette rejoint cette idée que l'autofiction est un privilège implicite qui hypostasie le récit fictionnel [...] en modèle de tout récit. ${ }^{30}$

L'autobiographie, langage de représentation doit toujours être fidele à une réalité présente, mettant l'accent sur la rencontre entre une intériorité et une extériorité. «Il y a dans l'autofiction l'idée que l'être ne pourra atteindre à sa vérité tant qu'il ne se sera pas élargi, mis en question par la fiction $»{ }^{31}$

Dans cette écriture où la fiction apparait comme un alibi de l'intime, le réel triomphe sur la fiction et se cache derrière elle.

Pour assumer explicitement cette responsabilité, d'être éloigné de tout mensonge, la narratrice se donne complètement à elle, à son autoréflexion pour émouvoir un monde magique de la littérature.

Un autre critère s'impose à l'autobiographie, genre délaissé depuis longtemps est une manifestation d'une nouvelle conception de la personne qui écrit son autobiographie.

Le fait d'écrire une intériorisation et une répression sociale qui amène au désir de révéler sa solitude; sa vie privée. Ainsi, recensé dans l'esprit humain; l'autobiographie moderne est un dévoilement de l'intériorité

Depuis le début du roman. K. Mourad ne cesse de revendiquer la problématique des enfants abandonnés qui ont le droit d'une autonomie individualiste et rejoint la ligne de Philippe Ariès dans son étude sur « L'enfant et la vie familiale sous "l'ancien Régime" qui montre que les différents " âges" de la vie ne sont pas une donnée naturelle mais sociale $»^{32}$ et que l'autobiographie témoigne de reprendre le mythe de l'enfance et de l'associer de manière flagrante à l'écriture autobiographique.

La narratrice, K. Mourad analyse dans son roman tous les aveux profonds d'une enfance désabusée vivant dans les tabous 
religieux (épisodes chez les religieuses), son convertisme au christianisme sans le vouloir; les interdictions; l'isolement au sein des familles adoptives; la privation du vrai amour adolescent et maturé, les répressions sexuelles accrues. Elle dessine de même tout un tableau significatif avec l'autre qui est mimé. Tout est décrit dans le moindre détail selon un acte de confession et de transgression qui rend sensible tout ce qui est interdit. Ainsi K. Mourad refait son texte et aménage les péripéties selon une mémoire active jouant sur les mécanismes d'une écriture où la parole quotidienne tient le premier rang par excellence. Par l'effet de la répétition, le lecteur poursuit avec ferveur qui rompt la monotonie, l'ordre des discours, des modulations de la voix, à tout ce que l'autobiographie lui dicte. Ce lecteur avisé partage ce qu'on lui inflige, alors que le niveau du langage de l'écrivaine comporte des choses qu'elle-même veut dévoiler et garde dans son for-intérieur des non-dits. A la limite, il ne faut pas croire que l'absence d'aveu et le silence dont il s'ensuit soit signe d'hypocrisie ou de refoulement.

La lecture de l'autobiographie (le « je » qui s'adresse aux lecteurs) implique un rapport d'intimité et de communication immédiate, acte révélateur, l'aveu devient un inconscient. Marcel Lobet affirme cette idée en disant :

«Le confesseur est avant-tout un " auditeur" recevant les aveux pour les interpréter dans le sens de cette chanté qui est un des noms de l'amour. Le critique écoute, non pour condamner, mais pour absoudre. Il ouvre son cœur autant que son esprit, il ne se montre sévère que devant les aberrations de l'original: l'absence du repentir ou l'obstination à confondre le bon et le mauvais ». ${ }^{33}$

Observatrice privilégiée d'elle-même, $\mathrm{K}$. Mourad (écrivaine faisant sa propre narration) rétablit les rapports humains dans leur transparence et dans leur véracité. Entre l'interdit et le désir; elle évoque une tentation de trouver l'aveu de la parole nostalgique de sa nature originaire. L'on voit bien que son récit autobiographique porte la marque de la résistance contre les tabous qui pèsent comme un fardeau lourd de significations inexplicables. A vrai dire, ce débat de circuit fermé tente à démystifier le récit autobiographique intéressant, riche de signes, susceptible d'amener des choses nouvelles.

Philippe Lejeune dans son article de revue littéraire comparative dit que: «l'aveu essaie de redonner le langage à un désir qui n'avait pas su s'exprimer: d'où la situation ambigüe du lecteur d'aveu, qui peut avoir le sentiment d'être là à la place de quelqu'un d'autre, mais qui, en même temps, sent se réveiller en lui tout ce qui aspire à la parole $\gg .^{34}$

L'écriture de K. Mourad dénote une narratrice omnisciente qui manipule l'effet de réel dans un jeu constant du mensonge et de la vérité.

Récit de crédibilité, l'autobiographie de K. Mourad est un genre à part mis au service d'une nécessaire vérité du texte et parvenant à persuader son lecteur. Ce genre qu'on qualifie d'être une littérature de "l'enchantement". Grâce à de multiples lectures critiques sur le récit autobiographique, il est clair que Zahr est un être vivant et non plus une simple figure de papier qui brise le pacte de lecture au fil des pages et se familiarise avec la narratrice errante.

Un élément imperceptible unit l'auteur à son récit. Le "je" avec ses variantes n'est autre que la voix même de l'écrivaine qui se définit dans une forme autobiographique particulière et substitue l'illusion du vrai par la vérité du vécu.

Cette apparition de l'auteur écrivaine émerge, tout le temps en premier lieu; saute à l'œil qui note, vérifie et analyse les détours. Cette apparition du nom "Zahr" tout au long du roman, jette une lueur particulière sur l'ensemble des récits narrés. Cette écrivaine humaniste, en premier lieu, pose en parallèle des questions philosophiques, religieuses et sociales 
inspirées par le drame de la situation. À maintes reprises, elle valorise les questions morales et éthiques, continue en parallèle d'écrire sa chronique de roman d'initiation à l'éducation enfantine à la manière de J.Jacques Rousseau dans l'Emile.

Dans les dernières pages du roman, le lien autobiographique résonne ici d'un écho sonore particulier dont la mort a précisément été annoncée dès l'incipit. Ce qui rend le schéma narratif, un schéma du type circulaire, et amène à une complexité croissante du récit. L'écart entre le narrateur - et l'écrivaine -personnage s'estompe par effet de répétition d'un "je" constant. L'on peut attribuer au roman de k.Mourad; le nom de pseudo-autobiographie ", dans lequel l'écrivaine décrit sa propre vie entière et peut- être le sujet d'une histoire ancrée dans le présent, à intervalles réguliers. L'espace ainsi se resserre entre le lecteur et le récit. Il ne s'agit pas d'une identification au héros, bien au contraire un éloignement involontaire; flottement des souvenirs mis en forme en vue de constituer l'innovation. Ici, en comparaison avec d'autres écrits autobiographiques, l'œuvre consiste à en apprécier les secrets intimes de l'écrivaine, et de l'énigme de son écriture.

Ch.I (1-3) Le revers irréversible (l'amour et la haine )

Comment pourrait - on dire des choses réelles avec des mots du cœur? L'auteure relate les étapes de son existence à la manière de J.J. Rousseau dans le sens de l'histoire d'une vocation. (Kenizé Mourad la personne réelle et la vie de son personnage fictif Zahr.) La difficulté de ce dédoublement qui accompagne le lecteur tout au long du roman ne relève point d'un narcissisme volontaire mais explicite une vision individualiste à la recherché d'une vérité subjective et un choix sélectif de l'expérience de vie et une conscience très fine .

L'écrivaine nous présente une nouvelle façade de l'autobiographie dans laquelle s'y mêlent mémoire, révolte, autofiction , témoignages . Le début ne subit pas une linéarité , la narratrice photographie et scrute sa vie par des fragments de mémoires "pris sur le vif".

La narration du début explique l'avènement de Zahr à Badalpour : le retour aux origines pour assister à la mort du père originaire le Radjah . Des partis de la narration qui s'amalgame avec le "je" torturé et souffrant sont conservés au journal intime, d'autres pour la mémoire surtout à l'âge enfantin, la jeunesse , les amis, l'école rappelant ainsi le souvenir de Nathalie Sarraute dans son roman Enfance, Gallimard, Paris , quand celle-ci souligne : «un enfant qui aime sa mère trouve que personne n'est plus beau qu'elle . Tout au long du récit autobiographique l'écrivaine ne cesse de se justifier, plaidoyer son projet d'écriture, se vide et s'en débarrasse du temps passé ( celui de l'isolement, l'abondance, la solitude d'un enfant souffre - douleur ). D'ailleurs sur soixante pages du grand volume romanesque évoquent avec amplitude et profondeur cette partie sinistre et refoulée entre amour et haine .

Faisant appel aux confessions de J.J. Rousseau elle interpelle un rôle d'une intertextualité nouvelle . Lorsque celui - ci âgé de dix ans mis en pension chez le Pasteur Lambercier à Bossey, près de Genève cite: «Je me rappelle toutes les circonstances des lieux, des personnes, des heures ». .35

A comparer cette citation avec celle évoquée par la narratrice heureuse, journaliste de quelques moments de joie où elle passait les vacances à Augen : "sitôt passé Augen à quelques km de Trintal, je ressuscitais (...) je considérais en effet Trintal comme mon fief inaliénable (...) pendant tout l'été, le village vivait à l'heure de garnements occupés à inventer les tours les plus pendables et nous nous retrouvions souvent perchés sur une branche .... ${ }^{36}$

$\mathrm{Ou}$ encore quand celle - ci entre dans le pensionnat religieux de la directrice MarieMarc: «Je me souviens d'une élève qui m'horripilait car elle récoltait toujours le cordon rouge et même la croix d'excellence 
.... Gersende de Sabran était ravissante (...) Mes amies de cœur étaient des moutons noirs, il y avait Annick une fille de divorcés (...) Par quel miracle était-elle entrée à l'institut Mirici d'où cette catégorie était impitoyablement rejetée ? Il y avait aussi Roselyn (...) fille de commerçants, $j$ 'aimais pourtant bien mes vieux parents, mais je ne m'y attachais pas vraiment, sachant n'être là que de passage : ma famille suisse , ma vraie famille, allait revenir me chercher. Je rêvais à ces retrouvailles j'en imaginais chaque détail . ${ }^{37}$

«Pour moi , l'amour était une faveur que l'on m'accordait, mais dont on me faisait bien comprendre que j'étais redevable (...) je n'eus pas à choisir: ils ne revinrent jamais me chercher ... Tout à coup, je n'existais plus . ${ }^{38}$

Pour s'abstenir contre la haine, l'écrivaine en se rappelant du moindre détail dans sa mémoire involontaire et fugitive essaye de relier les moments de joie instantanés pour faire volte face à une société qui n'accepte pas l'affectivité de ses émotions (amour ou haine ), l'écrivaine s'accroche à la négation et en utilisant les moments de silence de quarante ans, elle décide de révoquer sa mémoire comme une arme défensive contre la fuite du temps.

«Qu'on fait partie d'un tout et que jamais, jamais on ne peut-être seule?» ${ }^{39}$

«La vie était trop passionnante, je ne voulais pas en perdre une miette.» ${ }^{40}$

«C'est à Trintal qu'ont éclos tous mes rêves, c'est là (...) que j'ai appris le Bonheur.» $^{41}$

«Je n'étais plus une enfant abandonnée, j'avais un merveilleux papa, le plus merveilleux des papas.» ${ }^{42}$

«Je veux aller en Inde, moi je veux retrouver mon père $(. .$.$) désormais tout$ m'était égal.»>

Exprimant la haine en surcroît elle déclare «Tout le monde ne voulait que mon bien! Et , pour mon bien, sans le vouloir, ils tuaient tout ce qui en moi vivait. .

Ces quelques fragments soulignent le sentiment d'amertume et de haine d'une enfant innocente réfutée par la société et ses proches.

De multiples péripéties augmentent ce sentiment de haine mélangé par l'amour qui finit par une victoire triomphale. A la fin du roman, nous retrouvons Zahr gagnant le procès de son jardin, se donnant complètement à ses nièces ; elle leur relègue cet héritage précieux et disparut sans traces .Le roman se clôt par une série de questions sans réponses afin de prévaloir la libération de Zahr de ses fantômes.

«... ou bien, à présent qu'elle s'est libérée de ses fantômes, Zahr serait - elle simplement partie vers d'autres horizons légères, enfin prête à danser sa vie ? ${ }^{44}$

La clôture de la fin signale bien que Zahr après tant de monstruosités accidentelles qui planaient sur sa vie. Enfin «Zahr a fermé les yeux et savoure ce moment de bonheur. Mais elle sait aussi que, très loin des crispations délétères sur ce que je suis « et "ce que tu n'es pas », elle vit la des minutes de vérité qu'il lui faut goûter intensément pour ne pas les oublier $\gg .{ }^{45}$

\section{Ch.II (1-1) Le rôle de la mémoire}

L'ouvrage de k.Mourad, Le jardin de Badalpour est une collection de souvenirs passé d'enfance où chaque épisode constitue un fragment cristallisé instantané, arraché d'un passé nostalgique de l'écrivaine qui cherche tout le temps, le retour à un paradis perdu et disparu. Chaque tableau, qu'elle cite, la connaissance des familles dont elle a été fille adoptive, prend la forme d'un microcosme propre à l'écrivaine.

La description, avec beaucoup d'assiduité, provoque une vie en miniature. Non seulement, elle évoque le passé de Zahr mais également elle projette sur scène son secret utopique d'une histoire à la fois personnelle et mondiale.

Ouvrage digne de l'expérience exprimée, qui donne infiniment une richesse sur le plan textuel romanesque que sur le plan humain attesté.

Néanmoins, ce système d'écriture touché révélant un passé détruit par le scepticisme est en rapport avec sa propre expérience 
originaire. En aucune occasion le lecteur ne sent une différence entre Zahr et l'écrivaine qui ne ment jamais, ni se cache derrière une façade imaginaire.

Le réel ainsi apparaît dans toute son ampleur et sa force. Le fait de raconter et de décrire son enfance naît probablement d'un pressentiment d'une mort tragique injustifiée. Rappelant que Kenizé Mourad a pu mentionner tous ses sentiments les plus approfondis envers sa mère quand elle avait écrit « De la part de la princesse morte » dont Le jardin de Badalpour n'est qu'une continuation autobiographique. Les deux œuvres sont liées par une corde indénouable à une faculté de l'imagination autofictionnelle. Derrière l'apparition de cette œuvre, se réalise des morceaux narratifs de manière imprévue, dénotant une écriture automatique et aisée. Derrière la narration, se dégage une femme philosophe, qui a expérimenté la vie avec tous ses peines et ses chagrins.

Sous le regard du souvenir; sa philosophie se mue en poésie mondiale. Privée de nationalité indienne, turque, française, suisse, Kenizé Mourad n'avait pas pu absolument rien du patriotisme; ce qui explique en quelque sorte l'effet négatif d'un besoin chaviré et d'un amour profond à l'égard de son origine.

Toute sa vie est marquée par l'errance et l'égarement d'un enfant abandonné, et ce sentiment l'accompagnera durant la période de jeunesse.

Pour réaliser son œuvre, croit-on que cette écrivaine a utilisé l'imagination créative pour déréaliser le factuel ? Ce fut pour cela que l'auteur se proposa de raconter sa propre histoire afin d'y enregistrer dans la mémoire de telle photographie instantanée qui se révèle fugitive. Cette double dimension de remémoration et de pressentiment ne s'accomplit que dans le souvenir.

- Cette tension que ce soit dans la description des tableaux familiaux ou dans la vie des personnages, ou dans la narration, le lecteur assiste à une aura du propre passé autobiographique qui est en train de se décomposer. Cet itinéraire qu'on ose qualifier par «chasse aux papillons» devient apparence en écriture.

Remontant ainsi aux sources mêmes du vécu, représente l'essence même du récit autobiographique. Son objectif était de montrer dans cette œuvre, de quelle manière, elle a connu cette formation transcendantale qui brise toute temporalité.

- L'analyse de cette technique reproductive de l'âme qu'est l'imagination se fonde sur la possibilité à travers le récit autobiographique. Il s'agit de reconstruire, dans le souvenir, la perception originale du vécu.

Toute la structure du roman s'attache au rôle du souvenir instantané, mémorial de la narratrice. Le récit narré est classé par intermittence; contenant un mélange entre narration personnelle et description des lieux disparates entre l'Inde; la France, la Suisse. A l'intérieur des épisodes prises au vif s'enchaînent les séquences narratives touffues les unes avec les autres sans nuire à la cohérence du récit authentique. Histoire bouleversante, celle de Zahr, une histoire qui émane d'un désir lointain irrécupérable pour poursuivre les traces d'une enfance abandonnée, délaissée par un père, le Radja, riche et puissant qu'elle n'a jamais connu qu'à l'âge adolescent. Dans un jeu de prolepse et d'analepse, le jeu de la mémoire convoqué sur l'écran du souvenir interprété, le récit autobiographique grouille d'obsessions et de fantasmes et reste distancié du passé.

Le "je "du reprouvée, revit, témoigne et avoue sans recul. Le fait de descendre au fond d'un "je", c'est refuser l'exclusion expulsion de la barbarie sur l'autre. L'autobiographie "le récit de vie" réévalue le poids de l'individu et c'est ce que fait $\mathrm{K}$. Mourad : elle ouvre un espace textuel ou entrent en dialogue et en conflit un tempérament, un caractère, un idéal du moi pour construire une personnalité qui doit convaincre et séduire. 
Prisonnière d'une psyché étrangère dont elle se sature; elle sacrifie son propre "moi" emblématique et se crée écho et identification. C'est ainsi qu'elle se nie dans une forme douteuse d'abord masquée puis à visage découvert pour apparaître un personnage de plein droit à une vie.

L'intolérance à l'anonymat est un projet primordial dans l'œuvre. K. Mourad voulait sortir de l'invisibilité, être identifiée et accéder à la reconnaissance. Dans son récit autobiographie bouillonnent les désirs d'altération, attractions vers l'autre; pression externes, qui favorisent l'hybridation des épisodes, ce qui rend la perplexité de l'autobiographie un constant retour au personnel.

Ainsi le récit autobiographique trahit l'écriture et s'explicite dans une agressivité latente: sorte de libération d'un cri refoulé qui s'éclate. Le réel et le factice seront donc deux faces d'une même mémoire, d'une même identité.

De lourdes dissertations que le récit luimême, découvre le sens de la vie et l'histoire culturelle des enfants abandonnés, blessés de l'injustice sociale et sur la question que soulève $\mathrm{K}$. Mourad dans son œuvre. Pour clarifier cette pression de l'autobiographie, on pouvait dessiner un idéal d'évaluation d'une forme à l'autre: sentiment inavoué, suggéré, insinue d'analogie, projection de soi au miroir de l'autre d'un roman réflexif vers un " je" de l'autobiographie.

Les trajectoires effectives abondent en aller et retour selon des schéma-type. (les personnages qu'elle rencontre- le type de la même- du père - les religieuses - le jeune home qu'elle aimait - la tante -les familles qu'elle a dû rencontrer les amies de classe les confidentes- les maîtresses, réels ou fictifs, les personnages de K. Mourd sont des doubles qui gravitent autour de leur "auteur".

La narratrice questionne et commente leur action principale à peine démasquée par le sous-titre roman. Avec Zahr, la même protagoniste K. Mourad proclame d'emblée son registre exclusif qui la ramène à sa propre enfance .Ce roman de la quête errante tourne autour d'un "moi" omniprésent et tyrannique.

Pour accréditer son projet d'écriture personnelle, l'auteur réel (le moi) finit par admettre l'immanence de l'autobiographie élégante et subtile. Dans ce jeu scriptural de fictions délicieuses et déceptives, $\mathrm{K}$. Mourad exprime un égo qui aspire à se dire à travers ses personnages. Euvre composite, dynamique, l'œuvre abolit toute obligation et favorise une intimité virtuellement interminable.

La quête, les interrogations qui abondent dans le roman s'identifient au "je" qui ne cesse de vivre et de revivre. C'est un "je" qui s'impose et s'expose qui interpelle les secrets de l'âme. Ainsi la narratrice qui écrit son histoire devient possédée mais qui éprouve à la fois le tourment et la transfiguration. ${ }^{46}$

Pourquoi le recours à l'écriture de l'autobiographie ? Ou quelles sont les motivations de l'écriture autobiographique selon l'écrivaine?

Pour une simple raison, cette question qui s'impose au lecteur envisage la manière dont nous pourrions poser la question au besoin d'écrire au sein d'une philosophie autofictionnelle. Dans un tel recours, l'appel à s'exprimer, s'exposer, finit par passer à l'acte. Or, il s'avère que le lecteur implique bon gré, mal gré, dans le récit des vies que lui expose la narratrice.

Comme l'écrit Judith Butler dans le Récit de soi : "Le soi que j'ai encore à être ne peut que ridiculiser doucement la "posture du contrôle narratif". Je ne veux pas dire que je ne peux pas raconteur mon histoire mais que je ne peux faire autrement que spéculer. Ce que certains appellent autofiction et qui est une façon de perdre le fil du récit. Récit d'une conversion: comment, d'autre que j'étais, je suis devenu moi- même. Mais aussi histoire d'une reconnaissance: autre que j'étais sans possibilité de redevenir ce que j'étais. Manifestement, j'arrive trop tard à moi-même. C'est une aberration jusqu'à 
dans l'aberration, c'est - à - dire le devenir autre, maître de mon autobiographie $»^{47}$

Partant ainsi du récit de soi, il nous est apparu d'évoquer les propos de F. Nietzche «Faire usage de moi n'est pas seulement une injonction tendancieuse (abusive) c'est un fantasme $» .{ }^{48}$

En ce sens comme Althusser, dépossédé de son histoire mais maître de son récit $\mathrm{K}$. Mourad dirait hautement « Voilà ce que j'ai fait, ce que j'ai pensé, ce que je fus ». « $\mathrm{Ce}$ que j'ai compris ou cru comprendre (...), ce dont je ne suis plus tout à fait le maitre mais ce que je suis devenu $» .{ }^{49} \mathrm{Ou}$ encore le fait de rappeler : "Connais-toi toi-même en toi et fais-le savoir» «pourquoi ce que je suis pour moi, en moi, n'est pas ce que je suis pour les autres ? ${ }^{50}$

\section{ChII (1-2) La révolte le l'enfance}

La perception de l'enfant chez Kenizé Mourad, représente la possibilité même du récit autobiographique dans la mesure de sa constitution intime. Elle remonte même aux sources du vécu avec une pureté de vision qui s'établit sur sa capacité de fixer l'événement historique avant sa disparition.

Il conviendrait de lire l'autobiographie enfantine comme des aperçus originaux d'un « je » pur ou d'un sujet transcendantal afin de saisir une expérience supérieure qui se manifeste dans le champ du souvenir et de l'imagination. L'on ose dire que la narratrice se dissimule dans les mots et essaye de reconnaître des similitudes compulsives à devenir semblable aux autres et à se conduire comme eux.

C'est une contrainte qu'elle doit subir et n'arrive jamais à s'en débarrasser. Différente des autres; elle ne pouvait pas se rendre semblable aux modèles de moralité des autres, ni dans leurs attitudes et comportements même quand elle explique sa période de l'enfant à l'âge de cinq ans jusqu'à neuf, instruite par les religieuses ; elle éprouvait le sentiment d'une révolte irréparable.

Devenant si désemparée, elle ne pouvait même pas être ressemblante à elle-même, d'où le sentiment de vide et d'anéantissement. Dès lors, pour mieux saisir ce sens de la révolte pour soi et contre soi. K. Mourad avait une seule visée : c'est de reproduire avec honnêteté "l'image de soi-même » selon un processus de phénoménologie qui consiste à fonder un ancrage de représentation et de production d'images de cette enfance.

- Ainsi l'histoire racontée ne peut-être repassée dans le souvenir que sous la forme d'une histoire "imaginée". Ce fut pour cela que l'auteur - narratrice se propose de raconter sa propre histoire afin d'y enregistrer, une photographie instantanée.

M. Heidegger dans son «introduction à la métaphasique ${ }^{51}$ cite le modèle de ce phénomène comme étant «une enfance plongée dans un monde de fantasia axé sur la temporalité féerique propre à " ce qui n'est plus là " parce qu'il est ancré dans la matière du passé. » ${ }^{52}$

La faculté de reproduire le passé ou, en d'autres termes, l'acte imaginatif chez l'écrivaine est déterminé par un système de temporalisation assez particulier: c'est l'envie de capter et de posséder l'objet imaginé qui procure un certain bonheur à la narratrice ; car le sujet, rempli de réaliser à nouveau le passé s'avère un acte impossible qu'à travers le désir, le rêve de posséder ce qui a été perdu. Pour ceci; la mémoire autobiographique de K. Mourad tend à intégrer la couleur du temps sans $\mathrm{y}$ participer réellement. Cette constellation colorée d'idées; de rêve ; de désir de fuite du passé déclenche un système d'affinités dans le monde des choses qui n'existent plus. Entre mots- images-visions, l'écrivaine bâtit son autobiographie.

- N'oublions pas d'ajouter que K. Mourad a réussi, depuis sa formation de jeunesse à la quête de ses origines, d'intégrer l'ensemble dans un seul récit, l'idée d'amour et de haine.

Dès les premières pages, elle esquisse l'intégration de deux polarités contradictoires et uniques, l'amour et la haine à différents degrés sont incarnés dans un tourbillon de scènes colorées, reliées 
dans les fragments les uns aux autres d'une manière discontinuelle qui donne à une perplexité.

Dans l'ensemble de l'œuvre, Kenizé Mourad parvient à faire éclater la révolte pour l'amour et pour la haine dans un flux de mots redondants.

\section{Le symbole du jardin :}

«Badalpour, le berceau de la famille ou je me sens en paix, enfin chez moi après tant d'années d'errance». ${ }^{53}$

Contre toute haine, toute vengeance, le jardin constitue pour Zahr (la narratrice) et pour l'auteure la place du paradis perdu et révoqué; c'est le jardin de la sultane (sa mère).

«C'est au milieu du parc, un petit jardin clos d'une grille délicatement ouvragée, surmontée d'une plaque de marbre grave au nom de ma mère (...) c'est un jardin tout simple, sans plates - bandes ni fleurs rares ni arbres séculaires, seulement de la luzerne et des nuées de boutons - d'or au printemps, et, depuis quelques années, les jeunes manguiers que mon père et moi $\mathrm{y}$ avons plantés. Ce jardin est ma plus grande victoire (...) mon plus grand bonheur. Il est mon lien tangible avec l'Inde et Badalpour, il est surtout .... l'acte de reconnaissance de mon père. ${ }^{54}$

Lieu de rêves, de compensation; de reconnaissance, le jardin de Badalpour est un lieu cher marqué dans les souvenirs. Aggravé dans l'esprit de K. Mourad, il est tout le bonheur irremplaçable et le trésor qu'elle garde de ses origines murées dans la solitude qui grouille d'obsession et de fantasmes, le jardin convoqué sur l'écran de la description du souvenir revendique tout le passé de l'auteure.

K. Mourad en évoquant l'importance du jardin (d'ailleurs c'est le titre de son roman) témoigne et sollicite la sympathie du lecteur et la faveur du public littéraire; elle ouvre en cela un espace clos et ouvert (clos) en dimension, symbole de l'enfermement et ouvert ( pour la quête de l'identité - le bonheur et la libération) un espace textuel où entrent et sortent les dialogues - les conflits - l'idéal du moi pour reconstruire sa propre identité.

Lieu de conviction et de séduction par ses attraits et son caractère, la narratrice trouve le jardin, le seul lieu où elle s'identifie soi même dans une forme claire-obscure afin d'affirmer sa renaissance. Il convient de signaler que «Vivre, c'est être un autre ». ${ }^{55}$

Symbole du jardin, Lieu nostalgique :

Il existe en Inde, dans l'enceinte du palais le défunt Radjah de Badalpour, un petit jardin ou une jeune occidentale vient régulièrement se ressourcer, car Zahr, fille de sultane et descendante d'un des dernier souverains de Constantinople et fille de Radjah, et donc de cette terre indienne.

Son histoire est celle d'une fille qui en venant au monde a tout perdu son nom, son prénom, son âge, son pays, ses parents, sa mère ayant fui l'Inde l'a confiée avant de mourir à une famille adoptive.Une fois adulte, Zahr se lance dans la quête désespérée de sa véritable identité sans laquelle elle a l'impression de ne pouvoir commencer à vivre.

A vingt et un ans, après bien des années de recherches, elle retrouvera son père et son pays. Elle découvrira une famille d'échue depuis l'indépendance de l'Inde, une communauté musulmane minoritaire et persécutée. Ses habitudes d'occidentale choquent et lui valent maintes rebuffades.

En outre, elle n'est qu'une femme et, à ce titre n'a guère de droits. Mais elle a enfin trouvé le bonheur d'avoir une famille et un père qu'elle adule jusqu'a ce que son univers s'écroule à nouveau et qu'elle soit obligée de tout quitter.

Vingt ans plus tard elle revient et après bien des luttes, finit par comprendre que ces appartenances auxquelles chacun s'accroche, ne sont en fait que des béquilles qui aident à tenir debout, des barrières qui limitent, souvent même des caillères qui aveuglent. A présent libérée de ses fantômes, Zahr pourra $-\mathrm{t}$ - elle partir vers d'autres horizons, légère enfin prête à danser sa vie ? ${ }^{56}$ 


\section{Le type du colonisateur :}

Le fait d'écrire que la colonisation est terrible il n'en manque pas d'évoquer ses moyens terrifiants. Faisant allusion, à maintes reprises, aux représailles du colonisateur, K. Mourad nous parle de la pratique de l'occupant anglais qui terrorisait les peuples surdominés. Le refus révolté de ces peuples était un refus d'adhérer aux valeurs occidentales ainsi Zahr s'exclame: «Les Anglais voulaient introduire chez les peuples qu'ils dominaient leurs idéaux et leurs valeurs, leur apporter la bonne parole ; ils les laissaient vivre comme ils l'entendaient mais cela parce qu'ils les méprisaient, ainsi le colonisateur s'efforça à les christianiser, de les civiliser, de les transformer à leur image pour leur accorder la liberté après avoir pillé leur terre et leurs richesses. » ${ }^{57}$

Ainsi la narratrice triomphe par l'écriture sur toute sorte d'oppression et d'injustice sociale parce que dit-elle : «Si les choses ne passent pas par les entrailles, on fait de mauvais livres, les personnages, qu'ils soient fictifs ou historiques, il faut se les réapproprier, il faut les recréer. De cette sorte Zahr est une fille combattante, courageuse, fragile mais docile non seulement un personnage vraisemblable mais c'est Kenizé Mourad la vraie personne en chair et en os qui explore une dimension nouvelle de l'identité qui parait pour elle « une goutte d'eau contre le torrent, et c'est désespérant.» ${ }^{58}$

Ch II (1-3) Le phénomène de la mortification

De la part de la princesse morte, roman dans lequel K. Moura déplore la mort de sa mère, descendante d'une dynastie ottoman est révoquée encore dans le jardin de Badalpour. Le fait de pleurer la mère on le rencontre aussi chez Derrida qui avait les chances de se lire lui même de façon répétitive; cet événement se répète de façon performative, occurrente dans les souvenirs de la narratrice-auteur. Victime de cette mort, l'auteur ne cesse d'évoquer les souvenirs de la mère à chaque instant de douleur et de malheur.

«Je n'avais que six ans j'étais déjà profondément atteinte "d'abandonnite aiguë $\gg .59$

«J'avais honte. Honte de n'être somme toute qu'une "pauvre orpheline"». 60

«Etait-ma faute si ma maman était morte, si mon papa m'avait oubliée ? Etait-ce ma faute si les autres me prenaient quelque temps, mais sans jamais me garder? ${ }^{61}$

«Je n'eus pas à choisir: ils ne reviennent jamais me chercher $\gg .{ }^{62}$

«(...) Poussée par je ne sais quel instinct irrépressible je compris que c'était le visage de ma mère que je dessinais, ma mère que par la magie du trait, j'essayais de faire revivre imprimant son regard et son sourire sur tout ce qui m'entourait $\gg .{ }^{63}$

Quant au père, Zahr ou la narratrice évoque le seul souvenir de sa mort, « au bout de dix ans de silence, mon père se manifestait à nouveau ! ${ }^{64}$

«Il était à nouveau le séduisant Radjah de Badalpour, le père encore jeune que $j$ 'avais connu à l'âge de vingt et un ans et que j'avais tant aimé et tant haï ». ${ }^{65}$

«Que ne donnerais-je pour le comprendre pour l'arracher à cet enfermement qui est déjà la mort. Comment puis-je être sourde aux derniers mots de mon père, des mots venus du plus profond de lui, au moment où il se sait partir ? ... par peur d'avoir mal ... ou parce que j'ai envie, une envie monstrueuse de me venger ? ${ }^{66}$

Ainsi, K. Mourad éprouvait envers le père des sentiments de haine, de vengeance combinés avec des sentiments d'amour. Trouvant son père dès son retour dans un semi-coma, elle pleure son père à sa manière de mutisme et de douleurs en une série de questions qui forme un soliloque inouïe.

«Que suis- je au juste en train de faire? Est- ce que vraiment je lui donne la vie ou est-ce que je l'étouffe? Révulsée, les larmes aux yeux, je maintiens de force le masque en dépit de ses dénégations de plus en plus 
faibles. Comment puis-je le torturer ainsi $? »{ }^{67}$

« Il n'était plus le vieillard agonisant veillé par sa fille éplorée, il était à nouveau le séduisant Radjah de Badalpour, le père encore jeune que j'avais connu à l'âge de vingt et un ans et que $\mathrm{j}$ 'avais tant aimé et tant haï ».

La perplexité des sentiments de haine et d'amour est un système que la narratrice se procure pour aviver sa mémoire de chaque détail de l'autobiographie.

Ainsi décrivant son déchirement intérieur entre haine et amour combinés par les moments de silence et de stupéfaction elle déclare : «Il essayait de me dire, un mot qu'inconsciemment $\mathrm{j}$ 'attendais depuis trente ans mais aussi que je ne voulais pas entendre, un mot tout petit, mais pour nous deux immense : "Pardon". » ${ }^{68}$

Dans une suite de description des coutumes et habitudes appliquées en Orient, la description des scènes évoque la place de la religion islamique, les lieux du deuil, l'enterrement, les sentiments, les pleurs, le costume noir qu'on porte, le voile, le cercueil; tout un tableau y est présent avec la toile de fond d'un peintre, ainsi dit-elle : «Tour à tour, les hommes ont défilé, noirs et maigres dans leurs pauvres hardes, et avec avidité ils se sont penchés sur le visage de celui que jamais auparavant ils n'auraient osé regarder en face (...) tout farauds d'être en pleine santé, s'approchant jusqu'à examiner sous le nez ce géant enfin à leur portée (...) Rassurez-vous, Daddy, pas un n'a eu pitié»». ${ }^{69}$

En opposition à la description de cette scène; viennent l'expression des douleurs féminines lors de la mort d'un de ses proches. «Tante Zahra, jusqu'à-la si digne, se met à pousser de longs hurlements, déchirant ses voiles, se frappant les joues et le front tandis que mon frère Muzaffar s'écroule sur le sol en sanglotant, faisant mine de s'arracher les cheveux. $\gg^{70}$

La description du chapelet vient accentuer le mouvement. L'évocation de "le tesbih" ${ }^{71}$ est une marque du rituel islamique dans lequel on place le cercueil du défunt et on prie Dieu de racheter ses crimes et recevoir le salut dernier.

Dessinant ce rituel sacré, la narratrice n'y manque pas d'ajouter ses jugements et sa vision critique sur l'orient qu'elle s'enchérie. «Mais en Orient l'expression de la peine semble si peu naturelle, si codifiée. Peut-être est-ce que pour la canaliser, l'empêcher de déborder et de mettre ainsi en péril l'équilibre de la famille et de la société? $\gg^{72}$ Elle achève le tableau par une interrogation irrésolue en attendant émouvoir le sentiment du lecteur visé à apprécier ou dénoncer la description ainsi elle continue: "Comment évaluer le degré de force et de lucidité d'un être parvenu au seuil de la mort $\gg{ }^{73}$

\section{La mortification des autres :}

Le récit autobiographique de K. Mourad est mortifier aux autres: la mort du père et avant-elle, la mère sont convoquées dans des textes différents.

Evincé par ses proches dans des instants décisifs, l'auteur remet en cause toute relation à autrui. Son "moi" autobiographique n'a d'autre existence que celle que les autres lui donnent et lui renvoient.

Ce rapport aux autres (les familles adoptives) - (la famille d'origine) est stigmatisé par des impératifs imaginaires.

Du point du vue chronologique, c'est après la mort de la mère et du père que l'émergence de son moi s'affirme en vers et contre tout.

Le sentiment de revanche d'un enfant à qui la mort a volé la place. En figure d'écrivaine, elle décide de s'acquitter de ses dettes et de régler ses comptes par l'écriture autobiographique, afin de goûter l'injustifiable vie qu'elle mènera.

L'ambivalence autobiographique de $\mathrm{K}$. Mourad consiste à démentir ce qu'elle fut. Dans son statut figuratif d'écrivaine, l'auteure est consciente que l'autobiographie lui permet de se contredire à l'envie de transgresser à toute contradiction du pacte autobiographique. 
C'est pourquoi son récit est motivé par une duplicité ambigüe et discutable qui dépasse toute caractérisation comportementale ou psychologique pour désigner des modalités d'écriture qui disent le "soi".

Parler de soi, donc en se méfiant du langage, en refusant de faire confiance à l'écriture; en sachant qu'il est impossible de tout dire par parvenir à cette fin elle choisit de décrire les émotions du souvenir de la mère, du père, des amis, les désespoirs de toutes les familles qu'elle a dû rencontrées, les conditions de vie accablantes entre Orient et Occident, les existences sans perspectives d'avenir, de même elle n'évoque qu'accidentellement dans des fragments disloqués, la situation politique de l'Inde sous l'occupation britannique en 1939 symboliquement présentée en tant que figure de la patrie- mère.

Dans un projet de dénégation, de refus, l'écrivaine dénonce l'intolérance sociale, politique et religieuse c'est dans ce vécu subversif que l'écriture-là revêt un sens fidèle et conforme à la vérité.

En ce sens, comme critère de jugement et d'évaluation d'une dynamique scripturale, créative, revendique le contraire de toute forme de mensonge et de dissimulation, et confisque aux lecteurs tout déguisement autobiographique. «Mon pays c'est ma langue. L'identité, n'est ni le pays, ni la religion, ni même la famille. C'est le partage de valeurs essentielles avec des personnes. »

Dans cette exergue, k. Mourad s'engage dans l'écriture de son roman, Le jardin de Badalpour dans «une quête d'identité et dans la recherche de racines qui sont des préoccupations plus universelles que personnelles » (...). « Le chemin a été long et difficile car j'avais énormément de réticences qu'il me fallait vaincre et d'appréhensions à surmonter (...) d'aller si profondément en soi, d'explorer ainsi son terreau intérieur. C'était douloureux. Et j'estime que cela représente un grand acte d'humilité. Mon premier livre (indique-telle de la part de la princesse morte) est sans doute plus historique, plus chatoyant ; il fait rêver davantage. Mais le second (le jardin de Badalpour est à la fois plus universel et plus profond. Et quand j'ai fini, je ne me suis pas sentie délivrée, je me suis sentie vidée.» ${ }^{74}$

Portant le costume de l'occident, déchirée entre deux civilisations paradoxales, elle confronte la sévérité de respecter les traditions et son entourage ne la comprend jamais. Ce transport en Inde avec ses coutumes, sa population, ses palais, ses maharadjah, touche le cœur des lecteurs et les conduit à un flux émotionnel qui dépasse l'esprit.

Recherche émue, donc de la quête de soi, de l'identité rencontrant des personnages très différents, fait de l'expérience de ce parcours d'écrivaine, un manifeste d'appréciation de plus haut degré.

Dans ces deux mondes contradictoires, la narratrice trouve sa part de vérité partagée, si elle rejette un monde, elle accepte l'autre dans un mouvement de leitmotiv incessant complice au parcours.

Elle captive sa décision et au lieu de fuir la société dont laquelle elle s'introduise, elle essaye de la tolérer en la changeant. Imprégnée par son statut d'un enfant abandonné et délaissé, elle s'obstine à vouloir traiter tout le monde à un même pied d'égalité.

Symphonie poétique, l'essentiel est dit dans la présentation du roman plein de nouveaux attraits. L'intérêt, comme toute l'œuvre de l'auteure est de nous plonger dans une société pluridimensionnelle, avec ses codes sociaux notamment les rapports enfants / parents. Le roman s'offre sur les autres et sur leur environnement mélangé. La romancière connue dans toutes ces œuvres, s'y entremêle étroitement sur les croyances et fantasmes d'une époque, propre à elle seulement.

Décrivant l'évolution de sa vie, depuis sa naissance jusqu'à la trouvaille de ses origines. Un voyage de déception, de torture la poursuit tout au long du roman. Dans cette œuvre, on trouve déjà les grands 
thèmes du genre "autobiographie", opposition entre le bon et le mauvais, alliance matrimoniale et paternele contrariée, différents stades de racisme. Affrontements formidable entre légitimité et illégitimité pourchassant les humains. Dans ce roman, il n'est pas besoin de faire appel à l'imagination car Kenizé Mourad a fait un grand travail de description extraordinaire du fait réel.Serge Gainsbourg disait : «Quand on n'a pas ce que l'on aime, il faut aimer ce que l'on a. » septembre 1989.

Dans l'autobiographie, "narrateur" et "auteur" se confond; c'est la personne réelle qui existe, en chair ou en os et c'est celle qui assume la responsabilité de la production du texte. C'est elle qui organise le récit dont elle oriente la vision et choisit les voix des interlocuteurs. Entre narrateur et auteur, le récit autobiographique balance, entre le statut du narrateur et entre l'instance fictive intertextuelle qu'elle remplit.

Carole Tisset considère que le narrateur «n'existe que dans le texte. Il est la voix de papier qui raconte. Tandis que l'écrivain est extérieur au texte, même dans un récit autobiographique, le narrateur, lui, est dans le texte. Il n'est pas fait de chair et de sang mais constitué par deux monèmes essentiels : je ou il. ${ }^{75}$

Donc: c'est le narrateur impliqué qui faisant partie entre le personnage du monde fictionnel de l'œuvre et entre la personne de l'auteur réel qui fabrique le texte à sa guise ; qui participe aux événements déroulés en employant le «je». Personnage type « homodiégétique » selon les propres termes de Gérard Genette. ${ }^{76}$

Ici, dans le jardin de Badalpour, la narratrice souffre de l'anonymat mais toujours est omnisciente. Elle raconte deux événements ou plusieurs qui ont lieu en même temps dans deux espaces différents. La narratrice raconte l'histoire de Zahr qu'elle connait personnellement, puisqu'elle s'attribue le nom Zahr; c'est elle- même Kenizé Mourad qui a vécu l'histoire de Zahr dont elle connait les événements et les personnages parce qu'elle «a également le droit de commenter son histoire personnelle, ou les événements qu'il a vécus ${ }^{77}{ }^{77}$

\section{Conclusion}

Comme étant un produit scriptural sophistiqué, l'autobiographie de K. Mourad offre une diversité du vrai, du réel, du distrayant et du factuel.

Le "je" dynamique de l'autobiographie, torture le "moi" et le transforme de l'intérieur. Le récit autobiographique de $\mathrm{K}$. Mourad jette "poudre aux yeux", confirme autorité et personnalité du statut de l'écrivaine.

Artiste d'une exposition, elle a su confirmer sa propre existence d'une vie anonyme qui s'entame dans ses fantasmes.

Sujet, elle-même d'un alter ego, elle revendique dans son récit avec timidité parfois insolente, une pleine expression humaine autobiographique qui incarne une perplexité de notre temps. Répondant à une attestation forte d'une écrivaine créatrice, elle se dévoile originaire et ultime de la valeur du produit qu'elle exprime; "l'œuvre" autobiographique, ou l'écriture de soi".

$\mathrm{Ce}$ retour à l'origine, cette quête discontinuelle entre conversations, témoignages d'une vie vécue, ou qui semble être vécue grossièrement, fait apparaître l'écrivaine comme une analyste et mémorialiste, actrice de la vérité d'une vie. L'autobiographie culminerait dans les "vies" qu'elle s'imagine et se soumettrait aux imaginaires de son public.

Liée à l'absence de l'amour, à la haine et la mort, appel désespéré qui se noie dans une polyphonie de discours emboités, d'histoires mélancoliques, la vie de Zahr ou de K. Mourad est un instrument de séduction sur l'insistance du souvenir, le ressassement de la remémoration et la subjectivité avec toutes ses exigences et ses revendications.

Contre toute imagination K. Mourad plaide la cause du «vrai» et de «l'intime» et atteint en fin de compte la réalisation de son autobiographie.

Pour conclure, il nous convient de citer les mots de Judith Butler dans le Récit de soi: « Rien ne m'y oblige, absolument rien mais 
je commence dans le vif du sujet, je m'aperçois vite en vérité, quand bien même je m'autorise à le croire, que je ne suis pas l'auteur des conditions de ma propre émergence.» ${ }^{78}$

L'autobiographie de K. Mourad, œuvre perplexe contradictoire entre réel et fiction, n'est qu'une simple invitation à un art de vivre la vie dans la vérité, ou en d'autres termes selon Michel Foucault: « Des exercices... qui font partie de ce que nous pourrions appeler une "esthétique de soi" 》 ${ }^{79}$.

Le jardin de Badalpour est un roman autobiographique, un usage du "moi", une reconnaissance à l'autre, un renoncement à être l'auteur qui s'engage pour les autres mais qui a recours au vécu dans un processus de modelage du "soi".

\section{Références bibliographiques}

Nous recensons dans cette bibliographie; les œuvres de base sur l'autobiographie comme genre littéraire. Nous utilisons seulement, les œuvres critiques de base romanesque concernant le sujet proposé, et que nous avons utilisé seulement dans la recherche.

\section{Le corpus:}

Le jardin de Badalpour, ed.de Poche écrit en 1998 et 16 mai 2000. Coll, Fayard.

\section{Autres œuvres de Kenizé Mourad :}

- De la part de la princesse morte, ed. Le livre de Poche, 5 mai 1989, écrit en 1987.

- Le parfum de notre terre, ed. Robert Laffont, Paris, 2003.

- Dans la ville d'or et d'argent, ed. R. Laffont, 2016.

- A la rencontre des Maharanis, reines et princesses indiennes (12 livres).

- L'Inde au temps du Raj.

- Turquie (9livres).

- Nostalgie et splendeur au pays d Ataturk (26 livres).

Ouvrages généraux sur l'autobiographie:

- Goldmann, (Lucien), pour une sociologie du roman, Gallimard, Paris, 1965.

- Jean Starobinski, La relation critique, Paris, Gallimard, «Le chemin », 1970, p.90.
- Friedrich Nietzsche, généalogie de la morale, œuvres philosophiques complètes, VII, Paris, Gallimard, 1971.

- Georges, May: L'autobiographie, P.U.F, Paris, 1979.

- Aragon, Louis, Le Mentir - Vrai, Paris, Gallimard, 1980 réed. Folio no.3001.

- Le jeune (Philippe), Je est un autre, l'autobiographie de la littérature aux médias, Paris, Seuil, coll. ( poétique ) 1980 .

- Hamon (Philippe), Analyse du descriptif, Hachette, Paris, 1981.

- Hamon (Philippe), Le personnel du roman, ed. Drozo, Paris, 1983.

- Le jeune (Philippe), Moi aussi, Paris, ed. du seuil, coll. Poétique, 1986, p.21.

- Gaulejac (Vincent de) la névrose de classe: trajectoire social et conflits d'identité, Paris, Editions Hommes et groupes, 1987.

- Proust Marcel, A la recherche du temps perdu, ed. dirigée par Jean- Yves Tadié, Paris, Gallimard. "Bibliothèque de la Pléiade), T.111, 1988 p. 583.

- Serge Doubvrosky, Autobiographiques: de Corneille à Sartre, Paris, PUF, « perspectives critiques », 1988, p.72.

- W. Benjamin, Ecrits autobiographiques, Paris, Christian Bourgois, Éditeur, 1990.

- Jouve (Vincent), La lecture, Hachette, Paris, 1993, L'effet personnage dans le roman, ed. PUF, 1992, Paris.

- Sheringham Michael, French Autobiography: Devices and Desires. Rousseau to Perec. Oxford. Clarendon Press, 1993.Camus Albert, Le premier homme, posthume, Paris, Gallimard, 1994.

- Le jeune (Philippe), le pacte autobiographique, Paris Seuil, 1975, Edition: Nou. ed. augmentée, coll. «Points Essais », 1996.

- Le jeune (Philippe), Les Brouillons de soi, Paris, ed. du Seuil, 1998.

- Ruano - Borbalan ( Jean - Claude ). Coordonné par, L'identité: l'individu, le groupe, la société, Auxerre, sciences humaines, 1998. 
- Johan Faerber, Une vie sans histoire où l'impact autobiographique dans l'œuvre de Philippe Vilain.

- Lecarme, Jacques, Lecarme- Tabone, Éliane, L'autobiographie, Paris, Armand Colin, 1997.

- Louis Marin, l'écriture de soi, Paris, PUF, collège international de philosophie, 1999 p.114.

- Pierre Brunel, le romancier et ses doubles au XXème siècle, Paris, José Corti, 1997.

- Den Toonder, Jeannette M.L., Qui est je? l'écriture autobiographie et les nouveaux romanciers, Francfort - sur le Main, Peter Lang, 1999, "langue et littérature française », vol. 244.

- Françoise Simonet- tenant Maître de conférence de Littérature française Université Paris XIII, Le Journal intime, Nathan.

- Françoise Simonet, Genre Littéraire et écriture ordinaire, Nathan / Her, Paris, 2001.

- Michel Foucault, l'herméneutique du sujet. Cours au collège de France, 1981 1982, Paris, Gallimard, Le seuil, 2001 p. 31.

- W. Benjamin, Fragments philosophiques, politiques, critiques, littéraires, Christophe Jouanlanne et Jean-François Poirier, Paris, PUF, 2001, p. 122.

- Barthes (Roland), Fragments d'un discours amoureux œuvres complètes. Livres, textes, entretiens, tome IV, Paris, ed. du Seuil, 2002, p.577.

- Hubier Sébastien, Littératures intimes. Les expressions du moi de l'autobiographie à l'autofiction, Armand Colin / VUEF, 2003.

- Lejeune Philippe et Bogaret Catherine, Histoire d'une pratique, Un journal à soi, les éditions textuelles, septembre 2003.

- Bernard Stiegler, De la misère symbolique, La catastrophe du sensible, Paris, Galilée 2005, p. 181.

- Marcel Lobet, la ceinture de feuillage, essai sur la confession déguisée, Bruxelles, la Renaissance du livre, 1966 P. 14
- Miraux Jean-Philippe, L'autobiographie, Ecriture de soi et sincérité, ed. Armand Colin, 2005, 2007 pour la présente impression.

- Philippe Aries, l'enfant et la vie familial sous l'ancient regime, ed. Plon, Paris, 1960.

- Claude Arnaud, Qui dit je en nous? Une histoire subjective de l'identité, Paris, Grasset, 2006.

- Judith Butler, Le Récit de soi, Paris, PUF, 2007 p.82.

- Louis Althusser, L'avenir dure longtemps suivi de Les Faits, Paris, stock - IMEC, 2007.

- Diane Meur, Les vivants et les ombres, Paris, Sabine Wespieser, 2007.

Revues littéraires spécialisées et consacrées à l'autobiographie :

- Revue de littérature comparée, Autobiographies no.325 Janvier - Mars 2008, ed. Didier ERT Dition directeurs P. Brunel et D.-H. Pageaux. Coll. Klincksieck. 6. Paris.

- Article de Pierre Brunel, «Introduction aux autobiographies » p. 7 a 23.

- Article de Marcel de Grève, «L'autographie, genre littéraire » p.23.

- Article de Thomas Regnier (présenté par Jeremie Grangé) « de l'autobiographie à l'autofiction: une généalogie paradoxale, p 33.

- Article de Yves Michel Ergal, «De l'autobiographie dans le roman moderne » p. 67.

- Le Débat, no.144, Mars - Avril 2007, p.29 et p.3 à 69.

- Littérature, no. 10 Mai 1973: la punition des enfants. Lecture d'un aveu de Rousseau.

- Annales, Juillet - Août 1974 p.1009 1022, sous le titre: «Le dangereux supplément ».

- Poétique, no.3 (1970) article de Jean Starobinski : «Le style de l'autobiographie », p.257-265.

- Revue d'histoire littéraire de la France, Novembre - Décembre 1975 article de Georges $\ll$ De 
l'autobiographie iniatique

l'autobiographie genre littéraire ». Anthologie et dictionnaires :

- «Autobiographie »: - dans Jean Pierre de Beaumarchais, Daniel Couty, Alain Rey, dictionnaire des littératures de langue française, Paris, Bordas, 1987, t.1, p.116.

- Dictionnaire : le dictionnaire des lettres françaises, Paris, librairie générale française, la Pochothèque 1988 p. 27.

- Dictionnaire de critique littéraire, Paris, Armand Colin, 1993.

- Fernando Pessora, je ne suis personne une anthologie, Paris, Christian Bourgeois - 1994

- Dictionnaire européen des lumières, Paris, ed. du Seuil 1997 p.119.

- Ecrire la vie, Paris, Gallimard, coll. Quarto, 2011.

- Encyclopédie de l'Agora, article de Vivian Rakov: " le rêve Angélique" avril 2012.

Revues littéraires et articles des journaux:

- « L'écrivain, en terrain minée », le Monde, 25 Avril, 1985.

- «Retours », L'autre journal, Avril, 1985.

- «Histoires », Autrement, no.69, Août, 1985.

- «Quelque chose entre l'histoire, la sociologie, la littérature », La quinzaine littéraire, no. 532, 1989.

- Article au moment de la fête des mères, Prima magazine, Mai, 1992.

- Vers un je transpersonnel, in autofictions et Gie, sous la direction de Serge Doubrovski, Jacques. Lecarme et Philippe Lejeune cahiers RJTM, no.6, Université de Paris X - Nanterre, centre de recherches interdisciplinaires sur les textes modernes, 1993.

- « Tout livre est un acte », Europe, no. 784-785, Août - Septembre 1994.

- «L'enfance et la déchirure, Europe, no. 798, Octobre 1995.

- «De l'autre côté du siècle, nouvelle revue française, no.550, Juin 1999.
- Tisset Carole, "Analyse Linguistique de la narration P. 10 - coll, campus Linguistique, ed. Sedes, Paris 2000.

- "Vivre pour se raconter, se raconter pour vivre » avec Vincent de Gaulejac, Revue international de psychologie, printemps 2000 vol.VI, no. 14.

- «Mise à distance », revue des deux mondes, Juillet - Août 2003.

- «Raisons d'écrire », Nottingham, French Studies vol 48 no.2, juin 2009.

- L' écriture comme un couteau, avec Frédéric - Yves Jeannet, Paris, stock, 2003, réed, Gallimard, coll. Folio, 2011.

- L'usage de la photo, en collaboration avec Marc Marie, Paris, Gallimard, coll. Folio, 2005.

- Les années, paris, Gallimard, coll. Folio, 2008

- L'autre fille, Paris, Nil éditions, coll. «Les affranchis », 2011.

- Regarde les lumières mon amour, Paris, Seuil, coll « Raconter la vie :, 2014.

- Aissaoui (Laétitia), De Sousa Myriam), « Etranger ici, étranger là-bas », le discours identitaire des jeunes issus de l'immigration en France. Synergies Monde no.5, 2008, Université de Rouen.

- Gaulejac (Vincent de), «Roman familial et trajectoire sociale », in le récit d'enfance en question (colloque de Nanterre, 1987, du Philippe Lejeune), no. 12 des cahiers de sémiotique textuelle (publidix, Université de Paris X).

- Golsorkhi (Damon), Huault (Isabelle), Pierre Bourdieu : Critique et réflexivité comme attitude analytique en sciences de gestion, no. 165, 2006, 6, Editions Lavoisier.

\section{Sites internet :}

- La socialisation: 1'homme en tant qu'être social, par Matt. Dernière mise à jour le 03 mai 2009, in http://www.skyminds.net

- http://evene.lefigaro.fr citation/mot.php.mot.julien-green.

- http://raconterlavie.fr/collection

- http://souvenirssouvenirs.blogspace.fr/18 95278/la-famille-duraton. 
- http://www.erudit.org/culture/nb1073421 /nb1118578/208.8ac.pdf

- http://www.lapresse.ca/arts/livres/201101 /14/01-44

- http://fr-fr.facebook.com/Kenizé Mourad.

1. Cf : le journal (L'orient littéraire no.117 2016 interview avec K. Mourad)

2. Propre terme qui apparait avec G.de Nerval dans Aurélie et Sylvie au XIXème siècle.

3. Entretien avec K. Mourad, "une vie romanesque et fascinante", dans un article dans l'Orient littéraire p.3.

4. CF : Walter. Benjamin, Fragments philosophiques, politiques, critiques, littéraires, traduit par Christophe Jouanlanne et Jean-François Poirier, Paris, PUF, 2001, PP 67-70.

5. L'orient littéraire no. 117, Mars 2016 www.l'orient littéraire.com/ article_ détails p. 2 .

6. Idem, p.12.

7. Philippe Lejeune, Moi aussi, Paris, ed. du Seuil ,coll. poétique, 1986, p.21.

8. K. Mourad, le jardin de Badalpour, p.62-63.

9. cf. G. Genette, Je est un autre, coll. Seuil, Paris, 1980.

10. Propos de Louis Marin, L'écriture de soi, Paris, PUF, « collège international de philosophie, 1999 p.114.

11. Cf : Zahr dans l'onomastique arabe veut dire fleur et Selma (origine turque) veut dire intact-provient de paix.

12. K.Mourad, De la part de la princesse morte, Tome I.

13. Cf : Norman O. Brown, Marcuse et de Laing sur leurs travaux sur la psychanalyse.

14. Cf : Encyclopédie de l'Agora (pour un monde durable, dossier angélisme), article de Vivian Rakov «le rêve angélique » par avril 2012.

15. Cf. Philippe Lejeune : «le Je est un autre » nous nous sommes inspiré du titre de l'œuvre de ce critique sur l'autobiographie.
16. K. Mourad, op.cit, p.79, le jardin de Badalpour.

17. K. Mourad, op. cit, p.87-88.

18. Rivara René, La langue du récit, introduction à la narratologie énonciative, op.cit, p.29.

19. Rivara René, ibid p.162.

20. K. Mourad, le jardin de Badalpour, p.62-63.

21. Ibid, p.161.

22. Bakhtine Mikhaïl, « Esthétique et théorie du roman », Gallimard, Paris, 1978, p.396.

23. op.cit p.93.

24. Lejeune Philippe, « Je est un autre », coll. « Poétique », ed. Seuil, Paris, 1980, p.10.

25. A voir Jouve. Vincent, « La poétique du Roman » ed. Sedes, Campus lettres, 1997, p.33.

26. Genette Gérard « Nouveau Discours du récit », ed. du Seuil, Paris, novembre 1983, p.50.

27. Rivara René, op. cit p.85.

28. Cf .J.J Rousseau " le contrat social" ; nous avons emprunté le sens du titre seulement.

29. Cf. Jean Starobinski, la relation critique, 1970, p.87. Auteur-critique sur la transparence et l'obstacle, 1957. (Ouvrage fleur sur l'autobiographie de J.J.Rousseau.)

30. Cf. Genette, Gérard, Fiction et diction, ed. du seuil, Paris, p.65, 1990.

31. Cf. article de Thomas Régnier dans une revue de littérature comparée, p.36, no.325. Janvier - Mars 2008, ed. Klincksieck, Paris. « De l'autobiographie à l'autofiction une généalogie paradoxale ».

32. Cf. Philippe Ariès, L'enfant et la vie familiale sous l'Ancien Régime, ed. Plon, Paris, 1960.

33. Marcel Lobet, La ceinture de feuillage, essai sur la confession déguisée, Bruxelles, La Renaissance du livre, 1966, p.14.

34. Cf. Philippe Lejeune, revue de littérature comparée, (1-3), 2008, RCL, 
p.49, no. 325, 82ème année, Paris, ed. Klincksieck.

35. J.J.Rousseau, Les Confessions, Gallimard , Paris . coll. Folio, 2012.

36. Le jardin de Badalpour, Kenizé Mourad, p.57.

37. op. cit, p.59

38. Ibid, p.55.

39. op. cit, p.59

40. Ibid, p.58.

41. Idem, p.58.

42. Idem, p. 63-67.

43. Idem, p.63-67

44. Le jardin de Badalpour, p.569.

45. Op. cit, p.566.

46. Cf. Claude Arnaud, Qui dit je en nous? Une histoire subjective de l'identité, Paris, Grasset, 2006, p.22. (Arnaud expose son expérience, ses mobiles et ses transferts dans cette œuvre.)

47. Judith Butler, Le Récit de soi, Paris, PUF, 2007, p.82.

48. Friedrich Nietzsche, généalogie de la morale, œuvres philosophiques complètes, VII, Gallimard, 1971.

49. Louis Althusser, L'Avenir dure longtemps suivi de Les Faits, Paris, Sleek/IMEC, 2007.

50. J.J.Rousseau , p.48, Les Confessions.

51. M. Heidegger, Introduction à la métaphysique, Paris, Gallimard, p.189.

52. Note: citation prise de M. Heidegger, l'imagination est la faculté de représentation au moment où l'objet n'est plus là.

53. Op. cit p.17.

54. Le prologue du roman p.23-24.

55. Fernando Pessora, Je ne suis personne. Une anthologie, Paris, Christian bourgeois, 1994, p.32.

56. Cf : http//www.fayard.fr/le jardin de Badalpour, site internet dont j'ai retiré toutes les informations concernant le jardin- http//prix du quai des orfeires.fr.- $\mathrm{http} / / \mathrm{les}$ anges de la vigne.com

57. Reportage avec K. Mourad en TV5 (youtube.com videoblob Dynastie Ottomane.

58. www.ed.magazine.fr

59. Le jardin de Badalpour, p.36.

60. op.cit p.43.

61. Idem p.45.

62. Idem p.54.

63. Idem p.59.

64. Op.cit p.60.

65. Op.cit p.17.

66. Op.cit p.16.

67. Op.cit p.17.

68. Op.cit p.18.

69. Op.cit p.21.

70. Op.cit p.19.

71. "Le tesbih": terme qui désigne le chapelet musulman dont chacun des quatre-vingt-dix neuf grain correspond à un attribut de Dieu.

72. Idem p. 19

73. Op.cit p.15.

74. Entretien avec K. Mourad dans l'Orient littéraire p.2.

75. Tisset Carole, « Analyse linguistique de la narration» p.10. Collections Campus linguistique, Editions Sedes, Paris, 2000.

76. Gérard Genette, « Figures III », collections poétiques, ed. du Seuil, Paris, 1972, p.252.

77. Rivarex René «La langue du récit, introduction à la narratologie énonciative » p. 156; coll, l'Harmattan, Paris, 2000.

78. Op.cit. Judith Butler, Le Récit de soi, Paris, PUF, 2007, p.82.

79. Michel Foucault, l'Herméneutique du sujet. Cours au collège de France, 19811982, Paris, Gallimard, Le Seuil, 2001, p.166 